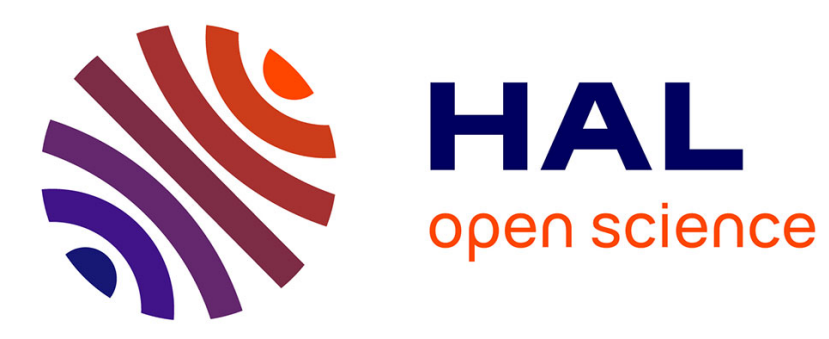

\title{
Visibly Pushdown Transducers with Well-nested Outputs
}

\author{
Pierre-Alain Reynier, Jean-Marc Talbot
}

\section{To cite this version:}

Pierre-Alain Reynier, Jean-Marc Talbot. Visibly Pushdown Transducers with Well-nested Outputs. 2014. hal-00988129

\section{HAL Id: hal-00988129 \\ https://hal.science/hal-00988129}

Submitted on 7 May 2014

HAL is a multi-disciplinary open access archive for the deposit and dissemination of scientific research documents, whether they are published or not. The documents may come from teaching and research institutions in France or abroad, or from public or private research centers.
L'archive ouverte pluridisciplinaire HAL, est destinée au dépôt et à la diffusion de documents scientifiques de niveau recherche, publiés ou non, émanant des établissements d'enseignement et de recherche français ou étrangers, des laboratoires publics ou privés. 


\title{
Visibly Pushdown Transducers with Well-nested Outputs
}

\author{
Pierre-Alain Reynier and Jean-Marc Talbot \\ Aix Marseille Université, CNRS, LIF UMR 7279, 13288, Marseille, France
}

\begin{abstract}
Visibly pushdown transducers (VPTs) are visibly pushdown automata extended with outputs. They have been introduced to model transformations of nested words, i.e. words with a call/return structure. When outputs are also structured and well nested words, VPTs are a natural formalism to express tree transformations evaluated in streaming. We prove the class of VPTs with well-nested outputs to be decidable in PTIME. Moreover, we show that this class is closed under composition and that its type-checking against visibly pushdown languages is decidable.
\end{abstract}

\section{Introduction}

Visibly pushdown automata (VPA) [1] (first introduced as input-driven pushdown automata [3]) are pushdown machines whose stack behavior is synchronized with the structure of the input word. More precisely, the input alphabet is partitioned into call and return symbols; when reading a call symbol the machine must push a symbol onto the stack, when reading a return symbol it must pop a symbol from the stack and when reading an internal symmbol the stack remains unchanged. Such words over a structure alphabet are called nested words.

Visibly pushdown transducers (VPTs) [7,8,6,9] extend visibly pushdown automata with outputs. Each transition is equipped with an output word; a VPT thus transforms an input word into an output word obtained as the concatenation of all the output words produced along a successful run (ie a sequence of transitions) on that input. VPTs are a strict subclass of pushdown transducers (PTs) and strictly extend finite state transducers. Several problems that are undecidable for PTs are decidable for VPTs similarly to finite state transducers: functionality (in PTIME), $k$-valuedness (in co-NPTIME) and functional equivalence (EXPTIME-complete) [6]. However, some decidability results or valuable properties of finite-state transducers unfortunately do not hold for VPTs: type-checking against VPA (deciding whether the range of a transducer is included into the language of a given VPA) is undecidable for VPTs and VPT are not closed under composition [7].

Unranked trees and more generally hedges can be linearized into nested words over a structured alphabet (such as XML documents). These words for which the matching between call and return symbols is perfect are called well-nested words. So, VPT are a suitable formalism to express hedge transformations. Moreover, as they process the linearization from left to right, they are also an adequate formalism to model and analyze transformations in streaming, as shown in [5]. VPTs output strings; operating on well-nested inputs, they define hedge-to-string transformations. If the output strings are well-nested too, they define hedge-to-hedge transformations [4]. 
In [6], by means of a syntactical restriction on transition rules, a class of VPTs whose range contains only well-nested words is presented. This class enjoys good properties: it is closed under composition and type-checking against visibly pushdown languages is decidable. One may then wonder whether these properties come from this particular subclass or from the fact that the ranges of these VPTs contain only wellnested words.

In this paper, we consider two classes of transductions (that is of relations) over nested words and definable by VPTs. First, the class of globally well-nested transductions, denoted $\mathcal{G}_{\mathrm{wn}}$, is the class of VPT transductions whose range contains only wellnested words. The second class, named almost well-nested and denoted $\mathcal{A}_{\mathrm{wn}}$, slightly generalizes the first one as follows: there should exist $k \in \mathbb{N}$ such that every output word contains at most $k$ unmatched returns, and at most $k$ unmatched calls. These two classes of transductions naturally define some classes of transducers gwnVPT and awnVPT, a VPT being in gwnVPT (resp. in awnVPT) if the transduction it represents is in $\mathcal{G}_{\mathrm{wn}}$ (resp. in $\mathcal{A}_{\mathrm{wn}}$ ). While defined in a semantical way, we provide criteria on successful computations of VPTs characterizing precisely the classes gwnVPT and awnVPT. Then based on these criteria, we prove the class awnVPT to be decidable in PSPACE. Regarding the class gwnVPT, using a recent result of [2], we prove it is decidable in PTIME. Finally, we prove that the two classes gwnVPT and awnVPT enjoy good properties: they are closed under composition and type-checking is decidable against visibly pushdown languages.

The paper is organized as follows: definitions and recalls of some basic properties on VPT are presented in Section 2. We introduce in Section 3 the two classes of transductions we define in this paper as well as the corresponding classes of transducers. Considering additionally the (restricted) class introduced in [6], we prove also that they form a strict hierarchy. Then, we give in Section 4 a precise characterization of the classes gwnVPT and awnVPT by means of some criteria on VPTs. Section 5 describes decision procedure of the considered classes of transducers. Finally, the closure of the considered classes under composition and the decidability of type-checking are addressed in Section 6. Omitted details can be found in the Appendix.

\section{Preliminaries}

(Well) nested words The set of all finite words (resp. of all words of length at most $n$ ) over a finite alphabet $\Sigma$ is denoted by $\Sigma^{*}$ (resp. $\Sigma^{\leq n}$ ); the empty word is denoted by $\epsilon$. A structured alphabet is a triple $\Sigma=\left(\Sigma_{c}, \Sigma_{i}, \Sigma_{r}\right)$ of disjoint alphabets, of call, internal and return symbols respectively. Given a structured alphabet $\Sigma$, we always denote by $\Sigma_{c}, \Sigma_{i}$ and $\Sigma_{r}$ its implicit structure, and identify $\Sigma$ with $\Sigma_{c} \cup \Sigma_{i} \cup \Sigma_{r}$. A nested word is a finite word over a structured alphabet.

The set of well-nested words over a structured alphabet $\Sigma$ is the least set, denoted by $\Sigma_{\mathrm{wn}}^{*}$, that satisfies $(i) \epsilon \in \Sigma_{\mathrm{wn}}^{*}$, (ii) for all $i \in \Sigma_{i}, w \in \Sigma_{\mathrm{wn}}^{*}, i w \in \Sigma_{\mathrm{wn}}^{*}$, and (iii) for all $w, w^{\prime} \in \Sigma_{\mathrm{wn}}^{*}, c \in \Sigma_{c}, r \in \Sigma_{r}, c w r w^{\prime} \in \Sigma_{\mathrm{wn}}^{*}$. E.g. on $\Sigma=\left(\left\{c_{1}, c_{2}\right\},\{r\}\right)$, the nested word $c_{1} r c_{2} r$ is well-nested while $r c_{1}$ is not.

For a word $w$ from $\Sigma^{*}$, we define its balance $\mathrm{B}$ as the difference between the number of symbols from $\Sigma_{c}$ and of symbols from $\Sigma_{r}$ occurring in $w$. Note that if $w \in \Sigma_{\mathrm{wn}}^{*}$, then $\mathrm{B}(w)=0$; but the converse is false as examplified by $r c_{1}$. 
Lemma 1. Let $u, v \in \Sigma^{*}$. We have $\mathrm{B}(u v)=\mathrm{B}(u)+\mathrm{B}(v)=\mathrm{B}(v u)$.

For any word $w$ from $\Sigma^{*}$, we denote by $\operatorname{Oc}(w)(\operatorname{resp} . \operatorname{Or}(w))$ the number of open calls (resp. open returns) in $w$. Formally,

$$
\operatorname{Or}(w)=-\min \left\{\mathrm{B}\left(w^{\prime}\right) \mid w^{\prime} w^{\prime \prime}=w\right\} \quad \operatorname{Oc}(w)=\mathrm{B}(w)+\operatorname{Or}(w)
$$

We define, for any word $w, \mathrm{O}(w)$ as the pair $(\operatorname{Or}(w), \operatorname{Oc}(w)) \in \mathbb{N}^{2}$. We also define $\|\mathrm{O}(w)\|=\max \{\operatorname{Or}(w), \operatorname{Oc}(w)\}$. Note that for any word $w, w \in \Sigma_{\mathrm{wn}}^{*}$ iff $\|\mathrm{O}(w)\|=0$, that is $\mathrm{O}(w)=(0,0)$.

Given a word $u \in \Sigma^{*}$, we define the height of $u$, denoted height $(u)$, as $\max \left\{\left\|\mathrm{O}\left(u_{1}\right)\right\| \mid\right.$ $\left.u=u_{1} u_{2}\right\}$. We denote by $|u|$ the length of $u$, defined as usual.

Definition 1. For any two pairs $\left(n_{1}, n_{2}\right)$ and $\left(n_{1}^{\prime}, n_{2}^{\prime}\right)$ of naturals from $\mathbb{N}^{2}$, we define $\left(n_{1}, n_{2}\right) \oplus\left(n_{1}^{\prime}, n_{2}^{\prime}\right)$ as the pair

$$
\left\{\begin{array}{l}
\left(n_{1}, n_{2}-n_{1}^{\prime}+n_{2}^{\prime}\right) \text { if } n_{2} \geq n_{1}^{\prime} \\
\left(n_{1}+n_{1}^{\prime}-n_{2}, n_{2}^{\prime}\right) \text { if } n_{1}^{\prime}>n_{2}
\end{array}\right.
$$

Proposition 1. $\left(\mathbb{N}^{2}, \oplus,(0,0)\right)$ is a monoid, and the mapping $\mathrm{O}$ is a morphism from $\left(\Sigma^{*}, ., \epsilon\right)$ to $\left(\mathbb{N}^{2}, \oplus,(0,0)\right)$; in particular, for any two words $u_{1}, u_{2}$ from $\Sigma^{*}, O\left(u_{1} u_{2}\right)=$ $O\left(u_{1}\right) \oplus O\left(u_{2}\right)$.

Transductions - Transducers Let $\Sigma$ be a structured (input) alphabet, and $\Delta$ be a structured (output) alphabet. A relation over $\Sigma^{*} \times \Delta^{*}$ is a transduction. We denote by $\mathcal{T}(\Sigma, \Delta)$ the set of these transductions. For a transduction $T$, the set of words $u$ (resp. $v$ ) such that $(u, v) \in T$ is called the domain (resp. the range) of $T$.

A visibly pushdown transducer from $\Sigma$ to $\Delta$ (the class is denoted $\operatorname{VPT}(\Sigma, \Delta)$ ) is a tuple $A=(Q, I, F, \Gamma, \delta)$ where $Q$ is a finite set of states, $I \subseteq Q$ the set of initial states, $F \subseteq Q$ the set of final states, $\Gamma$ the (finite) stack alphabet, and $\delta=\delta_{c} \uplus \delta_{r} \uplus \delta_{\iota}$ is the transition relation where:

- $\delta_{c} \subseteq Q \times \Sigma_{c} \times \Gamma \times \Delta^{*} \times Q$ are the call transitions,

- $\delta_{r} \subseteq Q \times \Sigma_{r} \times \Gamma \times \Delta^{*} \times Q$ are the return transitions.

- $\delta_{i} \subseteq Q \times \Sigma_{i} \times \Delta^{*} \times Q$ are the internal transitions.

A stack (content) is a word over $\Gamma$. Hence, $\Gamma^{*}$ is a monoid for the concatenation with $\perp$ (the empty stack) as neutral element. A configuration of $A$ is a pair $(q, \sigma)$ where $q \in Q$ and $\sigma \in \Gamma^{*}$ is a stack content. Let $u=a_{1} \ldots a_{l}$ be a (nested) word on $\Sigma$, and $(q, \sigma),\left(q^{\prime}, \sigma^{\prime}\right)$ be two configurations of $A$. A run of the VPT $A$ over $u$ from $(q, \sigma)$ to $\left(q^{\prime}, \sigma^{\prime}\right)$ is a (possibly empty) sequence of transitions $\rho=t_{1} t_{2} \ldots t_{l} \in \delta^{*}$ such that there exist $q_{0}, q_{1}, \ldots q_{l} \in Q$ and $\sigma_{0}, \ldots \sigma_{l} \in \Gamma^{*}$ with $\left(q_{0}, \sigma_{0}\right)=(q, \sigma),\left(q_{l}, \sigma_{l}\right)=\left(q^{\prime}, \sigma^{\prime}\right)$, and for each $0<k \leq l$, we have either $(i) t_{k}=\left(q_{k-1}, a_{k}, \gamma, w_{k}, q_{k}\right) \in \delta_{c}$ and $\sigma_{k}=\sigma_{k-1} \gamma$, or $(i i) t_{k}=\left(q_{k-1}, a_{k}, \gamma, w_{k}, q_{k}\right) \in \delta_{r}$, and $\sigma_{k-1}=\sigma_{k} \gamma$, or $(i i i)$ $t_{k}=\left(q_{k-1}, a_{k}, w_{k}, q_{k}\right) \in \delta_{i}$, and $\sigma_{k-1}=\sigma_{k}$. When the sequence of transitions is empty, $(q, \sigma)=\left(q^{\prime}, \sigma^{\prime}\right)$.

The length (resp. height) of a run $\rho$ over some word $u \in \Sigma^{*}$, denoted $|\rho|$ (resp. height $(\rho)$ ) is defined as the length of $u$ (resp. as the height of $u$ ). 
The output of $\rho$ (denoted output $(\rho)$ ) is the word $v \in \Delta^{*}$ defined as the concatenation $w=w_{1} \ldots w_{l}$ when the sequence of transitions is not empty and $\epsilon$ otherwise. We write $(q, \sigma) \stackrel{u \mid w}{\longrightarrow}\left(q^{\prime}, \sigma^{\prime}\right)$ when there exists a run on $u$ from $(q, \sigma)$ to $\left(q^{\prime}, \sigma^{\prime}\right)$ producing $w$ as output. Initial (resp. final) configurations are pairs $(q, \perp)$ with $q \in I$ (resp. with $q \in F$ ). A configuration $(q, \sigma)$ is reachable (resp. co-reachable) if there exists some initial configuration $(i, \perp)$ (resp. some final configuration $(f, \perp)$ ) and a run from $(i, \perp)$ to $(q, \sigma)$ (resp. from $(q, \sigma)$ to $(f, \perp)$ ). A run is accepting if it starts in an initial configuration and ends in a final configuration.

A transducer $A$ defines relation/transduction from nested words to nested words, denoted by $\llbracket A \rrbracket$, and defined as the set of pairs $(u, v) \in \Sigma^{*} \times \Delta^{*}$ such that there exists an accepting run on $u$ producing $v$ as output. Note that since both initial and final configurations have empty stack, $A$ accepts only well-nested words, i.e. $\llbracket A \rrbracket \subseteq$ $\Sigma_{\mathrm{wn}}^{*} \times \Delta^{*}$.

We denote $\mathcal{V P}(\Sigma, \Delta)$ the class of transductions defined by VPTs over the structured alphabets $\Sigma$ (as input alphabet) and $\Delta$ (as output alphabet).

Given a VPT $A=(Q, I, F, \Gamma, \delta)$, we let $\mathrm{O}_{\max }^{A}$ be the maximal number of open calls and of open returns in a word produced as output of a call or of a return transition in $A$. Formally, we have:

$$
\mathrm{O}_{\max }^{A}=\max \left\{\|\mathrm{O}(w)\| \mid(p, \alpha, w, \gamma, q) \in \delta_{c} \cup \delta_{r}\right\}
$$

Visibly pushdown automata We define visibly pushdown automata (VPA) simply as a particular case of VPT; we may think of them as transducers with no output. Hence, only the domain of the transduction matters and is called the language defined by the visibly pushdown automaton. For an automaton $A$, this language will be denoted $L(A)$.

Properties of computations in VPANPT We recall two standard results on runs of visibly pushdown machines.

Lemma 2. Let $A$ be a VPA with set of states $Q$ and $\rho:(p, \perp) \stackrel{u}{\rightarrow}(q, \perp)$ be a run of $A$ over some word $u \in \Sigma_{\mathrm{wn}}^{*}$. Let $h \in \mathbb{N}_{>0}$. We have:

(i) if height $(u)<h$ and $|u| \geq|Q|^{h}$, then $\rho$ can be decomposed as follows:

$$
\rho:(p, \perp) \stackrel{u_{1}}{\longrightarrow}\left(p_{1}, \sigma\right) \stackrel{u_{2}}{\longrightarrow}\left(p_{1}, \sigma\right) \stackrel{u_{3}}{\longrightarrow}(q, \perp)
$$

with $u_{1} u_{3}$ and $u_{2}$ well-nested words and $u_{2} \neq \epsilon$.

(ii) if height $(u) \geq|Q|^{2}$, then $\rho$ can be decomposed as follows:

$$
\rho:(p, \perp) \stackrel{u_{1}}{\longrightarrow}\left(p_{1}, \sigma\right) \stackrel{u_{2}}{\longrightarrow}\left(p_{1}, \sigma \sigma^{\prime}\right) \stackrel{u_{3}}{\longrightarrow}\left(p_{2}, \sigma \sigma^{\prime}\right) \stackrel{u_{4}}{\longrightarrow}\left(p_{2}, \sigma\right) \stackrel{u_{5}}{\longrightarrow}(q, \perp)
$$

with $u_{1} u_{5}, u_{2} u_{4}$ and $u_{3}$ well-nested words, and $\sigma^{\prime} \neq \perp$.

\section{Classes of VPT producing (almost) well-nested outputs}

In this section, after recalling the definition of (locally) well-nested VPT, we introduce the new classes of globally and almost well-nested VPT. Then, we prove relationships between these classes. 


\subsection{Definitions}

Locally Well-nested VPTs (IwnVPT) In [6], the class of (locally) well-nested VPT has been introduced. For this class, the enforcement of the well-nestedness of the output is done locally and syntactically at the level of transition rules.

Definition 2 (Locally Well-nested). Let $A=(Q, I, F, \Gamma, \delta)$ be a VPT. A is a locally well-nested VPT (IwnVPT) if:

- for any pair of transitions $\left(q, a, v, \gamma, q^{\prime}\right) \in \delta_{c},\left(p, b, w, \gamma, p^{\prime}\right) \in \delta_{r}$, the word $v w$ is well nested, and

- for any transition $\left(q, a, v, q^{\prime}\right) \in \delta_{i}$, the word $v$ is well-nested.

$A$ VPT transduction $T$ is locally well-nested if there exists a IwnVPT A that realizes $T$ $(\llbracket A \rrbracket=T)$. The class of locally well-nested $\mathrm{VPT}$ transductions is denoted $\mathcal{L}_{\mathrm{wn}}$.

It is straightforward to prove that

Proposition 2. Let $A$ be a locally well-nested VPT and $(p, \sigma),(q, \sigma)$ two configurations of $A$. For all well-nested word $u$, if $(p, \sigma) \stackrel{u / v}{\longrightarrow}(q, \sigma)$ then $v \in \Sigma_{\mathrm{wn}}^{*}$.

Therefore, any locally well-nested VPT transduction $T$ is included into $\Sigma_{\mathrm{wn}}^{*} \times \Delta_{\mathrm{wn}}^{*}$.

Globally well-nested VPT transduction - Almost well-nested VPT transduction In this section, we introduce the class of globally well-nested transductions and its weaker variant of "almost" well-nested transductions. Unlike the definition of $\mathcal{L}_{\text {wn }}$ which is done at the level of transducers, these definitions are done at the level of transductions and thus, as a semantical property.

Definition 3 (Globally Well-nested). A VPT transduction $T$ is globally well-nested if $T\left(\Sigma_{\mathrm{wn}}^{*}\right) \subseteq \Delta_{\mathrm{wn}}^{*}$. The class of globally well-nested VPT transductions is denoted $\mathcal{G}_{\mathrm{wn}}$.

$A$ VPT $A$ is globally well-nested if its transduction $\llbracket A \rrbracket$ is. The class of globally well-nested $\mathrm{VPT}$ is denoted gwnVPT.

Definition 4 (Almost Well-nested). A VPT transduction $T$ is almost well-nested if there exists $k$ in $\mathbb{N}$ such that for every pair of words $(u, v) \in T$, it holds that $\|\mathrm{O}(v)\| \leq$ $k$. The class of almost well-nested VPT transductions is denoted $\mathcal{A}_{\mathrm{wn}}$.

$A$ VPT $A$ is almost well-nested if its transduction $\llbracket A \rrbracket$ is. The class of almost wellnested $\mathrm{VPT}$ is denoted awnVPT.

\subsection{Comparison of the different classes}

Classes of transductions $\mathcal{G}_{\mathrm{wn}}$ and $\mathcal{A}_{\mathrm{wn}}$ are defined by semantical conditions on the defined relations. This yields a clear correspondence between the classes $\mathcal{G}_{\mathrm{wn}}$ and gwnVPT on one side and $\mathcal{A}_{\mathrm{wn}}$ and awnVPT on the other side. This is not the case for $\mathcal{L}_{\mathrm{wn}}$ : two examples of VPTs are given in Figure 1. It is easy to verify that $A_{1}, A_{2} \in$ gwnVPT. Moreover, none of these transducers belongs to IwnVPT. However, one can easily build a transducer $A_{2}^{\prime}$ such that $\llbracket A_{2} \rrbracket=\llbracket A_{2}^{\prime} \rrbracket$ and $A_{2}^{\prime} \in$ IwnVPT. Indeed one can perform the following modifications: 


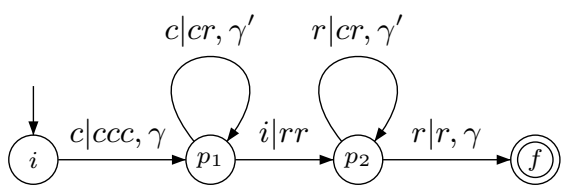

(a) The VPT $A_{1}$.

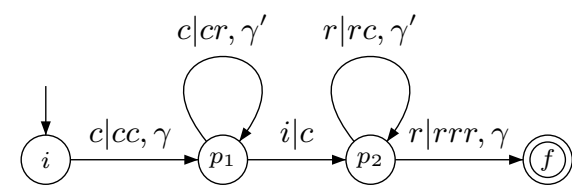

(b) The VPT $A_{2}$.

Fig. 1. Two VPTs in $\mathcal{V} \mathcal{P}(\Sigma, \Sigma)$ with $\Sigma_{c}=\{c\}, \Sigma_{r}=\{r\}$ and $\Sigma_{i}=\{i\}$.

- the transition $\left(p_{1}, i, c, p_{2}\right)$ becomes $\left(p_{1}, i, \epsilon, p_{2}\right)$

- the transition $\left(p_{2}, r, r c, \gamma^{\prime}, p_{2}\right)$ becomes $\left(p_{2}, r, c r, \gamma^{\prime}, p_{2}\right)$

- the transition $\left(p_{2}, r, r r r, \gamma, f\right)$ becomes $\left(p_{2}, r, c r r r, \gamma, f\right)$

On the contrary, as we prove below, the transduction $\llbracket A_{1} \rrbracket$ does not belong to $\mathcal{L}_{\mathrm{wn}}$ : there exists no transducer $A_{1}^{\prime} \in$ IwnVPT such that $\llbracket A_{1}^{\prime} \rrbracket=\llbracket A_{1} \rrbracket$.

To summarize, we prove the following proposition.

Proposition 3. The following inclusion results hold:

- For transducers: IwnVPT $\subsetneq$ gwnVPT $\subsetneq$ awnVPT

- For transductions: $\mathcal{L}_{\mathrm{wn}} \subsetneq \mathcal{G}_{\mathrm{wn}} \subsetneq \mathcal{A}_{\mathrm{wn}}$

Proof (Sketch). The non-strict inclusions are straightforward. The strict inclusions gwnVPT $\subsetneq$ awnVPT and $\mathcal{G}_{\mathrm{wn}} \subsetneq \mathcal{A}_{\mathrm{wn}}$ follow from the constraint on the range. The strict inclusion IwnVPT $\subsetneq$ gwnVPT is witnessed by $A_{2}$ from Figure 1, as explained above.

We sketch now the proof of the strict inclusion $\mathcal{L}_{\mathrm{wn}} \subsetneq \mathcal{G}_{\mathrm{wn}}$, and therefore consider the transducer $A_{1}$ on Figure 1. Observe that $\llbracket A_{1} \rrbracket \in \mathcal{G}_{\mathrm{wn}}$, we show that $\llbracket A_{1} \rrbracket \notin \mathcal{L}_{\mathrm{wn}}$. First note that $\llbracket A_{1} \rrbracket=\left\{\left(c c^{k} i r^{k} r, c c c(c r)^{k} r r(c r)^{k} r\right) \mid k \in \mathbb{N}\right\}$ and that

(Fact 1) the transduction defined by $A_{1}$ is injective

(Fact 2) any word of the output can be decomposed as $w_{1} r r w_{2}$ where $w_{1}=c c c(c r)^{k}$ and $w_{2}=(c r)^{k} r$ for some natural $k$ and for each $w_{1}$ with fixed $k$ there exists a unique $w_{2}$ such that $w_{1} r r w_{2}$ is in the range of $A_{1}$ (and conversely).

By contradiction, suppose that there exists $A_{1}^{\prime} \in \operatorname{IwnVPT}$ such that $\llbracket A_{1}^{\prime} \rrbracket=\llbracket A_{1} \rrbracket$. Now, for $k$ sufficiently large and depending only on the fixed size of $A_{1}^{\prime}, A_{1}^{\prime}$ has an accepting run for the input $c c^{k} i r^{k} r$ of the form given in the point (ii) of Lemma 2. Let us denote by $u_{i}$ (resp. $v_{i}$ ), $i \in\{1, \ldots, 5\}$ the corresponding decomposition of the input (resp. output) word. Due to Proposition 2, words $v_{1} v_{5}, v_{2} v_{4}$ and $v_{3}$ are well-nested.

Now, assume that $v_{2}=\epsilon$ and $v_{4}=\epsilon$. Then, using a simple pumping argument over the pair $\left(u_{2}, u_{4}\right)$, one would obtain a different input producing the same output, contradicting the injectivity of $A_{1}^{\prime}$ (due to (Fact 1)). So, $v_{2} \neq \epsilon$ or $v_{4} \neq \epsilon$.

Using a case analysis on the presence of the previously mentioned pattern $r r$ in the outputs of $A_{1}^{\prime}$, using the fact that $v_{2} v_{4} \neq \epsilon$, (Fact 2) and a pumping argument over the pair of words $\left(u_{2}, u_{4}\right)$, one obtains a contradiction. 


\section{Characterizations}

In this section we give criteria on VPTs that aim to characterize the classes gwnVPT and awnVPT.

Definition 5. Let A be a VPT. Let us consider the following criteria:

(C1) For all states $p, i, f$ such that $i$ is initial and $f$ is final, for any stack $\sigma$, then any accepting run

$$
(i, \perp) \stackrel{u_{1} / v_{1}}{\longrightarrow}(p, \sigma) \stackrel{u_{2} / v_{2}}{\longrightarrow}(p, \sigma) \stackrel{u_{3} / v_{3}}{\longrightarrow}(f, \perp)
$$

with $u_{1} u_{3}, u_{2} \in \Sigma_{\mathrm{wn}}^{*}$ satisfies $\mathrm{B}\left(v_{2}\right)=0$.

(C2) For all states $p, q, i, f$ such that $i$ is initial and $f$ is final, for any stack $\sigma, \sigma^{\prime}$, then any accepting run

$$
(i, \perp) \stackrel{u_{1} / v_{1}}{\longrightarrow}(p, \sigma) \stackrel{u_{2} / v_{2}}{\longrightarrow}\left(p, \sigma \sigma^{\prime}\right) \stackrel{u_{3} / v_{3}}{\longrightarrow}\left(q, \sigma \sigma^{\prime}\right) \stackrel{u_{4} / v_{4}}{\longrightarrow}(q, \sigma) \stackrel{u_{5} / v_{5}}{\longrightarrow}(f, \perp)
$$

with $u_{2} u_{4}, u_{3} \in \Sigma_{\mathrm{wn}}^{*}$ and $\sigma^{\prime} \neq \perp$ satisfies $\mathrm{B}\left(v_{2}\right)+\mathrm{B}\left(v_{4}\right)=0$ and $\mathrm{B}\left(v_{2}\right) \geq 0$.

It holds that

Theorem 1. Let $A$ be a VPT. Then $A$ is almost well-nested iff $A$ verifies criteria $\left(C_{1}\right)$ and $\left(C_{2}\right)$.

This is an immediate consequence of Propositions 4 and 5 that we prove now.

Lemma 3. Let $X \subseteq \Sigma^{*}$ such that the set $\mathrm{B}(X)=\{\mathrm{B}(u) \mid u \in X\}$ is infinite. Then the set $\{\mathrm{O}(u) \mid u \in X\}$ is infinite as well.

Lemma 4. Let $u \in \Sigma^{*}$ and $k$ be a strictly positive integer. Then $\mathrm{O}\left(u^{k}\right)$ is equal to $(\operatorname{Or}(u),(\operatorname{Oc}(u)-\operatorname{Or}(u)) *(k-1)+\operatorname{Oc}(u))$ if $\operatorname{Oc}(u) \geq \operatorname{Or}(u)$ and to $(\operatorname{Or}(u)+$ $(\operatorname{Or}(u)-\operatorname{Oc}(u)) *(k-1), \operatorname{Oc}(u))$ otherwise.

Proof. By definition of $\oplus$ and by induction on $k$.

Proposition 4. Let $A$ be a VPT. If $A$ does not satisfy $(C 1)$ or $(C 2)$, then $A$ is not almost well-nested.

Proof. Let us assume that $A$ does not satisfy $(C 1)$. Hence there exists an accepting run as described in criterion $(C 1)$ such that $\mathrm{B}\left(v_{2}\right) \neq 0$. We then build by iterating the loop on word $u_{2}$ accepting runs for words of the form $u_{1}\left(u_{2}\right)^{k} u_{3}$ for any natural $k$, producing output words $v_{1}\left(v_{2}\right)^{k} v_{3}$. Let us denote this set by $X$. As $\mathrm{B}\left(v_{2}\right) \neq 0$ and by Lemma 1, the set $\mathrm{B}(X)$ is infinite. Lemma 3 entails that $A$ is not almost well-nested.

Assume now that $A$ does not satisfy $(C 2)$. Hence, there exists an accepting run as described in the statement of the proposition such that either $(i) \mathrm{B}\left(v_{2}\right)+\mathrm{B}\left(v_{4}\right)=b \neq 0$ or $(i i) \mathrm{B}\left(v_{2}\right)<0$. In the case of $(i)$, from this run, one can build by pumping accepting runs for words of the form $u_{1}\left(u_{2}\right)^{k} u_{3}\left(u_{4}\right)^{k} u_{5}$ for any natural $k$, producing output words $v_{1}\left(v_{2}\right)^{k} v_{3}\left(u_{4}\right)^{k} v_{5}$. As before, Lemmas 1 and 3 imply that $A$ is not almost well-nested. 
Now, for $(i i)$ assuming that $\mathrm{B}\left(v_{2}\right)+\mathrm{B}\left(v_{4}\right)=0$. $\mathrm{As} \mathrm{B}\left(v_{2}\right)<0$, it holds that $\mathrm{B}\left(v_{4}\right)>$ 0 and thus, $\operatorname{Or}\left(v_{2}\right)>\operatorname{Oc}\left(v_{2}\right), \operatorname{Or}\left(v_{4}\right)<\operatorname{Oc}\left(v_{4}\right)$. From the run of the statement, one can build by pumping accepting runs for words of the form $u_{1}\left(u_{2}\right)^{k} u_{3}\left(u_{4}\right)^{k} u_{5}$ for any natural $k$, producing output words $v_{1}\left(v_{2}\right)^{k} v_{3}\left(v_{4}\right)^{k} v_{5}$. Now, we consider $\mathrm{O}\left(v_{1}\left(v_{2}\right)^{k} v_{3}\left(v_{4}\right)^{k} v_{5}\right)$ which, by associativity of $\oplus$, is equal to $\left.\mathrm{O}\left(v_{1}\right) \oplus \mathbf{O}\left(\left(v_{2}\right)^{k}\right) \oplus \mathbf{O}\left(v_{3}\right) \oplus \mathbf{O}\left(\left(u_{4}\right)^{k}\right) \oplus \mathbf{O}\left(v_{5}\right)\right)$. Now, by Lemma 4, it is equal to

$$
\begin{array}{r}
\mathrm{O}\left(v_{1}\right) \oplus\left(\operatorname{Or}\left(v_{2}\right)+\left(\operatorname{Or}\left(v_{2}\right)-\operatorname{Oc}\left(v_{2}\right)\right) *(k-1), \operatorname{Oc}\left(v_{2}\right)\right) \oplus \mathrm{O}\left(v_{3}\right) \oplus \\
\left(\operatorname{Or}\left(v_{4}\right),\left(\operatorname{Oc}\left(v_{4}\right)-\operatorname{Or}\left(v_{4}\right)\right) *(k-1)+\operatorname{Oc}\left(v_{4}\right)\right) \oplus \mathrm{O}\left(v_{5}\right)
\end{array}
$$

It is easy to see that for $k$ varying, the described pairs are unbounded.

Given a VPT $A=(Q, I, F, \Gamma, \delta)$, we define the integer $N_{A}=2|Q|^{2|Q|^{2}}$.

Lemma 5. Let $A$ be a VPT. If $A$ satisfies the criteria $(C 1)$ and $(C 2)$, then for any accepting run $\rho$ such that $|\rho| \geq N_{A}$, there exists an accepting run $\rho^{\prime}$ such that $\left|\rho^{\prime}\right|<|\rho|$ and $\| \mathrm{O}\left(\right.$ output $\left.\left(\rho^{\prime}\right)\right)\|\geq\| \mathrm{O}($ output $(\rho)) \|$.

Proof (Sketch). Let $A=(Q, I, F, \Gamma, \delta)$ and $\rho$ be an accepting run such that $|\rho| \geq N_{A}$. We distinguish two cases, depending on height $(\rho)$ :

- when height $(\rho)<2|Q|^{2}$ : by definition of $N_{A}$, we can apply Lemma 2.(i) twice and prove that $\rho$ is of the following form:

$$
(i, \perp) \stackrel{u_{1} / v_{1}}{\longrightarrow}(p, \sigma) \stackrel{u_{2} / v_{2}}{\longrightarrow}(p, \sigma) \stackrel{u_{3} / v_{3}}{\longrightarrow}\left(q, \sigma^{\prime}\right) \stackrel{u_{4} / v_{4}}{\longrightarrow}\left(q, \sigma^{\prime}\right) \stackrel{u_{5} / v_{5}}{\longrightarrow}(f, \perp)
$$

with $u_{2}, u_{4} \in \Sigma_{\mathrm{wn}}^{*} \backslash\{\epsilon\}$. Then, by criterion $(C 1)$, we have $\mathrm{B}\left(v_{2}\right)=\mathrm{B}\left(v_{4}\right)=0$. One can prove that at least one of $u_{2}$ and $u_{4}$ can be removed from $u$ while preserving the value $\operatorname{Or}(u)$. Let us denote by $v^{\prime}$ the resulting output word. Observe also that removing this part of the run does not modify the balance $B($.$) of the run, as$ $\mathrm{B}\left(v_{2}\right)=\mathrm{B}\left(v_{4}\right)=0$. As $\mathrm{Oc}(v)=\mathrm{B}(v)+\operatorname{Or}(v)$, we obtain $\mathrm{O}(v)=\mathrm{O}\left(v^{\prime}\right)$, yielding the result.

- when height $(\rho) \geq 2|Q|^{2}$ : in this case, we can apply Lemma 2.(ii) twice and prove that $\rho$ is of the following form:

$$
\begin{aligned}
& (i, \perp) \stackrel{u_{1} / v_{1}}{\longrightarrow}\left(p_{1}, \sigma\right) \stackrel{u_{2} / v_{2}}{\longrightarrow}\left(p_{1}, \sigma \sigma_{1}\right) \stackrel{u_{3} / v_{3}}{\longrightarrow}\left(q_{1}, \sigma \sigma_{1} \sigma_{2}\right) \stackrel{u_{4} / v_{4}}{\longrightarrow}\left(q_{1}, \sigma \sigma_{1} \sigma_{2} \sigma_{3}\right) \\
& \stackrel{u_{5} / v_{5}}{\longrightarrow}\left(q_{2}, \sigma \sigma_{1} \sigma_{2} \sigma_{3}\right) \stackrel{u_{6} / v_{6}}{\longrightarrow}\left(q_{2}, \sigma \sigma_{1} \sigma_{2}\right) \stackrel{u_{7} / v_{8}}{\longrightarrow}\left(p_{2}, \sigma \sigma_{1}\right) \stackrel{u_{8} / v_{8}}{\longrightarrow}\left(p_{2}, \sigma\right) \stackrel{u_{9} / v_{9}}{\longrightarrow}
\end{aligned}
$$
$(f, \perp)$, with $u_{1} u_{9}, u_{2} u_{8}, u_{3} u_{7}, u_{4} u_{6}, u_{5} \in \Sigma_{\mathrm{wn}}^{*}$ and $\sigma_{1}, \sigma_{3} \neq \perp$.

Then the two following runs can be built: the one obtained by removing the parts of $\rho$ on $u_{2}$ and $u_{8}$, and the one obtained by removing the parts of $\rho$ on $u_{4}$ and $u_{6}$, yielding runs whose length is strictly smaller than $|\rho|$. Let us denote these two runs by $\rho^{\prime}$ and $\rho^{\prime \prime}$ respectively, and their outputs by $v^{\prime}$ and $v^{\prime \prime}$. As $A$ verifies the criterion $(C 2)$, we have that $\mathrm{B}(v)=\mathrm{B}\left(v^{\prime}\right)=\mathrm{B}\left(v^{\prime \prime}\right)$, as $\mathrm{B}\left(v_{2}\right)+\mathrm{B}\left(v_{8}\right)=\mathrm{B}\left(v_{4}\right)+\mathrm{B}\left(v_{6}\right)=0$ and $\mathrm{B}$ is commutative. In order to obtain the result, we study $\operatorname{Or}(v)$. Considering different cases, we manage to prove that either $\operatorname{Or}\left(v^{\prime}\right) \geq \operatorname{Or}(v)$ or $\operatorname{Or}\left(v^{\prime \prime}\right) \geq \operatorname{Or}(v)$. The result follows as for any word $w$ we have $\operatorname{Oc}(w)=\mathrm{B}(w)+\operatorname{Or}(w)$.

Proposition 5. Let $A$ be a VPT. If A satisfies $(C 1)$ and $(C 2)$, then every accepting run $\rho:(i, \perp) \stackrel{u \mid v}{\longrightarrow}(f, \perp)$ of $A$ verifies $\|\mathrm{O}(v)\| \leq N_{A} \cdot \mathrm{O}_{\max }^{A}$. 
Proof. If $|v| \leq N_{A}$ the result is trivial; otherwise, assuming the existence of a minimal counter-example of this statement, a contradiction follows from Lemma 5.

Now we can show a precise characterization of transducers from gwnVPT amongst those in awnVPT.

Definition 6. Let $A$ be a VPT. We consider the following criterion:

(D) For all $(u, v) \in \llbracket T \rrbracket$, if $|u| \leq N_{A}$ then $v \in \Sigma_{\mathrm{wn}}^{*}$.

Theorem 2. Let $A$ be a VPT. Then $A$ is globally well-nested iff $A$ verifies criteria $\left(C_{1}\right),\left(C_{2}\right)$ and $(D)$.

Proof. The left-to-right implication is trivial. The other one is an easy consequence of Lemma 5.

\section{Deciding the classes of almost and globally well-nested VPT}

In this section, we prove that given a VPT $A$, it is decidable to know whether $\llbracket A \rrbracket \in \mathcal{A}_{\mathrm{wn}}$ and whether $\llbracket A \rrbracket \in \mathcal{G}_{\mathrm{wn}}$.

It is known that

Proposition 6. The following problems can be solved in PTIME: given $A=(Q, I, F, \Gamma, \delta)$ $a$ VPT and states $p, q$ of $A$. Decide whether there exists some stack $\sigma$ such that:

- $(p, \sigma)$ is reachable

- $(q, \sigma)$ is co-reachable

- $(p, \sigma)$ is reachable and $(q, \sigma)$ is co-reachable

Theorem 3. Let $A$ be a VPT. Whether $\llbracket A \rrbracket \in \mathcal{A}_{\mathrm{wn}}$ can be decided in PSPACE.

Proof (Sketch). By Theorem 1, deciding the class awnVPT amounts to decide criteria $(C 1)$ and $(C 2)$. Therefore we propose a non-deterministic algorithm running in polynomial space, yielding the result thanks to Savitch theorem.

We claim that $A$ verifies $(C 1)$ and $(C 2)$ if and only if it verifies these criteria on "small instances", defined as follows:

- Criterion $(C 1)$ : consider only words $u_{2}$ such that height $\left(u_{2}\right) \leq|Q|^{2}$ and $\left|u_{2}\right| \leq$ 2. $|Q|^{|Q|^{2}}$.

- Criterion $(C 2)$ : consider only stacks $\sigma^{\prime}$ such that $\left|\sigma^{\prime}\right| \leq|Q|^{2}$ and words $u_{2}, u_{4}$ of

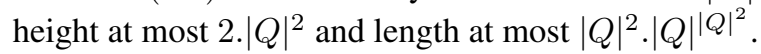

The non-deterministic algorithm follows from the claim: in order to exhibit a witness of the fact that $A \notin$ awnVPT, the algorithm guesses whether $(C 1)$ or $(C 2)$ is violated, and a pair of states $(p, q)$ and one or two runs, according to the criterion, of exponential size, which can be done in polynomial space. Using Proposition 6 it also verifies that there exists a stack $\sigma$ such that $(p, \sigma)$ is reachable and $(q, \sigma)$ is co-reachable.

To prove this claim, we show, by induction on $u \in \Sigma_{\mathrm{wn}}^{*}$, that for every run $(p, \perp) \stackrel{u \mid v}{\longrightarrow}$ $(q, \perp)$ that can be completed into an accepting run, and for every decomposition of this run according to criterion $\left(C_{1}\right)$ or $(C 2)$, the property stated by the corresponding criterion is fulfilled. 
The previous algorithm could be extended to handle in addition criterion $(D)$, yielding a PSPACE algorithm to decide whether a VPT $A$ is globally well-nested. However, we can use a recent result to prove that this problem can be solved in PTIME.

Theorem 4. Let $A$ be a VPT. Whether $\llbracket A \rrbracket \in \mathcal{G}_{\mathrm{wn}}$ can be decided in PTIME.

Proof. This proof heavily relies on results from [2] showing that deciding whether a context-free language is included into a Dyck language can be solved in PTIME.

We first erase the precise symbols of the produced outputs keeping track only of the type of the symbols: we build from $A$ a VPT $A^{\prime}$ defined on the output alphabet $\Sigma^{\prime}$ with $\Sigma_{c}^{\prime}=\left\{(\}, \Sigma_{r}^{\prime}=\{)\right\}$ and $\Sigma_{i}^{\prime}=\emptyset$. A transition of $A^{\prime}$ is obtained from a transition of $A$ by replacing in output words of the transition of $A$ call symbols by ( and return symbols by ) and removing internal symbols. It is then easy to see that $A$ is in gwnVPT iff $A^{\prime}$ is in gwnVPT (actually, for each run in $A$ producing $v$, its corresponding run in $A^{\prime}$ produces some $v^{\prime}$ such that $\mathrm{O}(v)=\mathrm{O}\left(v^{\prime}\right)$ ). Then, as shown in [8], one can build in polynomial time a context-free grammar $G_{A^{\prime}}$ generating the range of $A^{\prime}$. Finally, we appeal to [2] to conclude.

\section{Closure under composition and Type-checking}

\subsection{Definitions and existing results}

In this section, we consider two natural problems for transducers : the first one is related to composition of transductions. The second problem is the type-checking problem that aims to verify that any output of a transformation belongs to some given type/language. For VPT, the obvious class of "types" to consider is the class of languages defined by VPA.

Definition 7 (Closure under composition). A class $\mathcal{T}$ of transductions included in $\Sigma^{*} \times \Sigma^{*}$ is closed under composition if for all $T, T^{\prime}$ in $\mathcal{T}$, the transduction $T \circ T^{\prime}$ is also in $\mathcal{T}$. It is effectively closed under composition if for any transducers $A, A^{\prime}$ such that $\llbracket A \rrbracket, \llbracket A^{\prime} \rrbracket \in \mathcal{T}, A \circ A^{\prime}$ is computable and $\llbracket A \circ A^{\prime} \rrbracket$ is in $\mathcal{T}$.

A class of transducers $\mathrm{T}$ is effectively closed under composition iffor any two transducers $A, A^{\prime}$ in $\mathrm{T}, A \circ A^{\prime}$ is computable and $A \circ A^{\prime}$ is in $\mathrm{T}$.

Definition 8 (Type-checking (against VPA)). Given a VPT $A$ and two VPA $B, C$, decide whether $\llbracket A \rrbracket(L(B)) \subseteq L(C)$.

The following results give the status of these properties for arbitrary VPTs and for IwnVPT:

Theorem 5 ([7,6]). Regarding closure under composition, we have:

- The class $\mathcal{V P}(\Sigma, \Sigma)$ is not closed under composition.

- The class IwnVPT is effectively closed under composition.

In addition, the problem of type checking against VPA is undecidable for (arbitrary) VPT and decidable for IwnVPT. 


\subsection{New results}

Actually, regarding the closure under composition of the class IwnVPT, though it is not explicitly stated, the result proved in [6] is slightly stronger. It is indeed shown that for any VPT $A, B$ such that $A \in$ IwnVPT, there exists an (effectively computable) VPT $C$ satisfying $\llbracket C \rrbracket=\llbracket A \rrbracket \circ \llbracket B \rrbracket$. In addition, if $B \in$ IwnVPT, then $C \in$ IwnVPT.

We extend this positive result to any almost well-nested transducer.

One of the main ingredients of the proof of this result is the set $\mathcal{U P S} \mathcal{S}_{A}$ defined for any VPT transducer $A=\left(Q_{A}, I_{A}, F_{A}, \Gamma_{A}, \delta^{A}\right)$ as

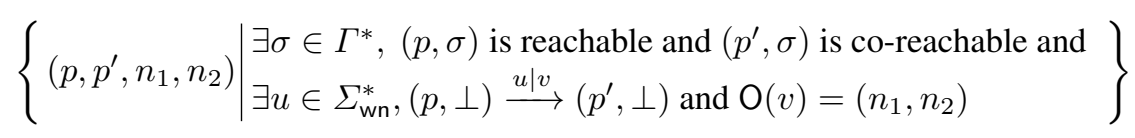

Proposition 7. Let $A$ in awnVPT. Then the set $\mathcal{U P S}_{A}$ is finite and computable in exponential time in the size of $A$.

Theorem 6. Let $A, B$ be two VPTs. If $A$ is almost-well nested, then one can compute in exponential time in the size of $A$ and $B$ a VPT $C$ such that $\llbracket C \rrbracket=\llbracket A \rrbracket \circ \llbracket B \rrbracket$. Moreover, if $B$ is also almost well-nested, then so is $C$, and if $A$ and $B$ are globally well-nested, then so is $C$.

Proof (Sketch). We present the construction of $C$, the proof of correctness can be found in the Appendix. By Proposition 7, $\mathcal{U P S} \mathcal{S}_{A}$ is finite and we let $K$ be the computable integer value $\max \left(\left\{\pi_{3}(x) \mid x \in \mathcal{U P} \mathcal{S}_{A}\right\} \cup\left\{\pi_{4}(x) \mid x \in \mathcal{U P} \mathcal{S}_{A}\right\}\right)$.

Given $B=\left(Q_{B}, I_{B}, F_{B}, \Gamma_{B}, \delta^{B}\right)$, we define $C=\left(Q_{C}, I_{C}, F_{C}, \Gamma_{C}, \delta^{C}\right)$ as

$$
\begin{array}{ll}
Q_{C}=Q_{A} \times Q_{B} \times \Gamma_{B}^{\leq K} & I_{C}=I_{A} \times I_{B} \times\{\perp\} \\
\Gamma_{C}=\Gamma_{A} \times \Gamma_{B}^{\leq \mathrm{O}_{\max }^{A}+K} & F_{C}=F_{A} \times F_{B} \times\{\perp\}
\end{array}
$$

Now for the transition rules $\delta^{C}$ :

- $\left((p, q, \sigma), i, w,\left(p^{\prime}, q^{\prime}, \sigma^{\prime}\right)\right) \in \delta_{i}^{C}$ if there exist a word $v \in \Delta^{*}$ and a stack $\sigma_{0} \in \Gamma_{B}^{*}$ such that $\sigma=\sigma_{0} \sigma_{1}, \sigma^{\prime}=\sigma_{0} \sigma_{1}^{\prime}, \mathrm{O}(v)=\left(\left|\sigma_{1}\right|,\left|\sigma_{1}^{\prime}\right|\right)$, and $\left(p, i, v, p^{\prime}\right) \in \delta_{i}^{A}$ and there exists a run $\left(q, \sigma_{1}\right) \stackrel{v \mid w}{\longrightarrow}\left(q^{\prime}, \sigma_{1}^{\prime}\right)$ in $B$,

- $\left((p, q, \sigma), c, w,\left(\gamma, \sigma_{3}\right),\left(p^{\prime}, q^{\prime}, \sigma_{4}\right)\right) \in \delta_{c}^{C}$ if there exist a word $v \in \Delta^{*}$, two stacks $\sigma_{0}, \sigma_{2} \in \Gamma_{B}^{*}$ and a stack symbol $\gamma \in \Gamma_{A}$ such that $\sigma=\sigma_{0} \sigma_{1}, \mathrm{O}(v)=\left(\left|\sigma_{1}\right|,\left|\sigma_{2}\right|\right)$, $\sigma_{0} \sigma_{2}=\sigma_{3} \sigma_{4},\left(p, c, v, \gamma, p^{\prime}\right) \in \delta_{c}^{A}$ and there exists a run $\left(q, \sigma_{1}\right) \stackrel{v \mid w}{\longrightarrow}\left(q^{\prime}, \sigma_{2}\right)$ in $B$, such a transition exists provided the bounds on the sizes of the different stacks are fulfilled, i.e. $|\sigma| \leq K,\left|\sigma_{4}\right| \leq K$, and $\left|\sigma_{3}\right| \leq \mathrm{O}_{\max }^{A}+K$

- $\left((p, q, \sigma), r, w,\left(\gamma, \sigma_{3}\right),\left(p^{\prime}, q^{\prime}, \sigma^{\prime}\right)\right) \in \delta_{r}^{C}$ if there exist a word $v \in \Delta^{*}$, a stack $\sigma_{0} \in$ $\Gamma_{B}^{*}$ such that $\sigma_{0} \sigma_{1}=\sigma_{3} \sigma, \sigma_{0} \sigma_{2}=\sigma^{\prime}, \mathrm{O}(v)=\left(\left|\sigma_{1}\right|,\left|\sigma_{2}\right|\right),\left(p, r, v, \gamma, p^{\prime}\right) \in \delta_{r}^{A}$ and there exists a run $\left(q, \sigma_{1}\right) \stackrel{v \mid w}{\longrightarrow}\left(q^{\prime}, \sigma_{2}\right)$ in $B$

such a transition exists provided the bounds on the sizes of the different stacks are fulfilled, i.e. $|\sigma| \leq K,\left|\sigma^{\prime}\right| \leq K$, and $\left|\sigma_{3}\right| \leq \mathrm{O}_{\max }^{A}+K$

Intuitively, in a state of $C$, we store the current states of $A$ and $B$. In addition, a part of the top of the stack of $B$ is also stored in the state of $C$ to allow the simulation of $B$. The (finite) amount that needs to be stored in the state is identified using the $\operatorname{set} \mathcal{U P S} \mathcal{S}_{A}$. 
Corollary 1. The classes $\mathcal{G}_{\mathrm{wn}}$ and $\mathcal{A}_{\mathrm{wn}}$ are (effectively) closed under composition.

Theorem 7 (Type-checking against VPA). Given an almost well-nested VPT $A$ and two visibly pushdown automata $B, C$, whether $\llbracket A \rrbracket(L(B)) \subseteq L(C)$ is decidable in 2 - EXPTIME.

Proof. Restricting the domain of $A$ to $L(B)$ is easy: it suffices to compute the product VPA of $A$ and $B$. Then, VPA being closed under complementation, we compute $\bar{C}$, the complement of $C$. Note that the size of $\bar{C}$ is at most exponential in the size of $C$. We then turn $\bar{C}$ into a transducer $C^{\prime}$ defining the identity relation over $L(\bar{C})$ (this is obvious by simply transforming rules of $\bar{C}$ into rules of transducers outputting their input). Now, by Theorem 6 , one can build a transducer defining the composition of $\llbracket A \rrbracket \circ \llbracket C^{\prime} \rrbracket$. This can be done in doubly exponential time in the size of $A$ and $C$. Now, it is sufficient to test whether the VPA underlying this transducer is empty or not.

\section{Conclusion}

In this paper, we have considered and precisely characterized the class of VPT with well-nested outputs. We have shown that this class is closed under composition and that its type-checking against VPA is decidable. We have restricted ourselves in this paper to transducers with well-nested domains. We conjecture that this restriction can be easily relaxed and thus, one could consider transducers based on nested word automata [1]. We left open the problem of deciding the class $\mathcal{L}_{\text {wn }}$. As we have described on some examples, this problem is far from being trivial. In [4], a clear relationship between the class IwnVPT and hedge-to-hedge transducers is described; investigating such a relationship for gwnVPT is also an interesting problem.

\section{References}

1. R. Alur and P. Madhusudan. Adding Nesting Structure to Words. JACM, 56(3):1-43, 2009.

2. A. Bertoni, C. Choffrut, and R. Radicioni. The inclusion problem of context-free languages: Some tractable cases. Int. J. Found. Comput. Sci., 22(2):289-299, 2011.

3. B. v. Braunmühl and R. Verbeek. Input-driven Languages are Recognized in log n Space. In FCT, volume 158 of $L N C S$, pages 40-51. Springer, 1983.

4. M. Caralp, E. Filiot, P.-A. Reynier, F. Servais, and J.-M. Talbot. Expressiveness of visibly pushdown transducers. In Proceedings Second International Workshop on Trends in Tree Automata and Tree Transducers, TTATT 2013, volume 134 of EPTCS, pages 17-26, 2013.

5. E. Filiot, O. Gauwin, P.-A. Reynier, and F. Servais. Streamability of Nested Word Transductions. In FSTTCS, volume 13 of LIPIcs, pages 312-324. Schloss Dagstuhl - Leibniz-Zentrum fuer Informatik, 2011.

6. E. Filiot, J.-F. Raskin, P.-A. Reynier, F. Servais, and J.-M. Talbot. Properties of Visibly Pushdown Transducers. In MFCS'10, volume 6281 of LNCS, pages 355-367. Springer, 2010.

7. J.-F. Raskin and F. Servais. Visibly pushdown transducers. In ICALP, volume 5126 of LNCS, pages 386-397, 2008.

8. F. Servais. Visibly Pushdown Transducers. PhD thesis, Université Libre de Bruxelles, 2011.

9. S. Staworko, G. Laurence, A. Lemay, and J. Niehren. Equivalence of deterministic nested word to word transducers. In FCT, volume 5699 of LNCS, pages 310-322, 2009. 


\section{A Proofs of Section 2}

Proof (of Proposition 1). We only prove that $\left(\mathbb{N}^{2}, \oplus,(0,0)\right)$ is a monoid. The second part of the statement is an easy consequence.

Let us prove that $(0,0)$ is a neutral element. Obviously, $\left(n_{1}, n_{2}\right) \oplus(0,0)=\left(n_{1}, n_{2}\right)$. Now, for $(0,0) \oplus\left(n_{1}^{\prime}, n_{2}^{\prime}\right)$, if the first case applies then $0 \geq n_{1}^{\prime}=0$ and the result is indeed $\left(0, n_{2}^{\prime}\right)=\left(n_{1}^{\prime}, n_{2}^{\prime}\right)$. Otherwise, the second case applies and gives $\left(n_{1}^{\prime}, n_{2}^{\prime}\right)$.

Now, let us prove that $\oplus$ is associative.

(1a) $\left(n_{1}, n_{2}\right) \oplus\left(n_{1}^{\prime}, n_{2}^{\prime}\right)=\left(n_{1}, n_{2}-n_{1}^{\prime}+n_{2}^{\prime}\right)$ if $n_{2} \geq n_{1}^{\prime}$.

Now, $\left(\left(n_{1}, n_{2}\right) \oplus\left(n_{1}^{\prime}, n_{2}^{\prime}\right)\right) \oplus\left(n_{1}^{\prime \prime}, n_{2}^{\prime \prime}\right)$ is equal to

$$
\left\{\begin{array}{l}
\left(n_{1}, n_{2}-n_{1}^{\prime}+n_{2}^{\prime}-n_{1}^{\prime \prime}+n_{2}^{\prime \prime}\right) \text { if } n_{2}-n_{1}^{\prime}+n_{2}^{\prime} \geq n_{1}^{\prime \prime}\left(\text { ie } n_{2}+n_{2}^{\prime} \geq n_{1}^{\prime}+n_{1}^{\prime \prime}\right) \\
\left(n_{1}+n_{1}^{\prime \prime}-n_{2}+n_{1}^{\prime}-n_{2}^{\prime}, n_{2}^{\prime \prime}\right) \text { if } n_{2}-n_{1}^{\prime}+n_{2}^{\prime}<n_{1}^{\prime \prime}
\end{array}\right.
$$

(1b) $\left(n_{1}, n_{2}\right) \oplus\left(n_{1}^{\prime}, n_{2}^{\prime}\right)=\left(n_{1}+n_{1}^{\prime}-n_{2}, n_{2}^{\prime}\right)$ if $n_{2}<n_{1}^{\prime}$.

Now, $\left(\left(n_{1}, n_{2}\right) \oplus\left(n_{1}^{\prime}, n_{2}^{\prime}\right)\right) \oplus\left(n_{1}^{\prime \prime}, n_{2}^{\prime \prime}\right)$ is to equal to

$$
\left\{\begin{array}{l}
\left(n_{1}+n_{1}^{\prime}-n_{2}, n_{2}^{\prime}-n_{1}^{\prime \prime}+n_{2}^{\prime \prime}\right) \text { if } n_{2}^{\prime} \geq n_{1}^{\prime \prime} \\
\left(n_{1}+n_{1}^{\prime}-n_{2}+n_{1}^{\prime \prime}-n_{2}^{\prime}, n_{2}^{\prime \prime}\right) \text { if } n_{2}^{\prime}<n_{1}^{\prime \prime}
\end{array}\right.
$$

(2a) $\left(n_{1}^{\prime}, n_{2}^{\prime}\right) \oplus\left(n_{1}^{\prime \prime}, n_{2}^{\prime \prime}\right)=\left(n_{1}^{\prime}, n_{2}^{\prime}-n_{1}^{\prime \prime}+n_{2}^{\prime \prime}\right)$ if $n_{2}^{\prime} \geq n_{1}^{\prime \prime}$.

Now, $\left(n_{1}, n_{2}\right) \oplus\left(\left(n_{1}^{\prime}, n_{2}^{\prime}\right) \oplus\left(n_{1}^{\prime \prime}, n_{2}^{\prime \prime}\right)\right)$ is equal to

$$
\left\{\begin{array}{l}
\left(n_{1}, n_{2}-n_{1}^{\prime}+n_{2}^{\prime}-n_{1}^{\prime \prime}+n_{2}^{\prime \prime}\right) \text { if } n_{2} \geq n_{1}^{\prime} \\
\left(n_{1}+n_{1}^{\prime}-n_{2}, n_{2}^{\prime}-n_{1}^{\prime \prime}+n_{2}^{\prime \prime}\right) \text { if } n_{2}<n_{1}^{\prime}
\end{array}\right.
$$

(2b) $\left(n_{1}^{\prime}, n_{2}^{\prime}\right) \oplus\left(n_{1}^{\prime \prime}, n_{2}^{\prime \prime}\right)=\left(n_{1}^{\prime}+n_{1}^{\prime \prime}-n_{2}^{\prime}, n_{2}^{\prime \prime}\right)$ if $n_{2}^{\prime}<n_{1}^{\prime \prime}$.

Now, $\left(n_{1}, n_{2}\right) \oplus\left(\left(n_{1}^{\prime}, n_{2}^{\prime}\right) \oplus\left(n_{1}^{\prime \prime}, n_{2}^{\prime \prime}\right)\right)$ is to equal to

$$
\left\{\begin{array}{l}
\left(n_{1}^{\prime}+n_{1}^{\prime \prime}-n_{2}^{\prime}+n_{1}-n_{2}, n_{2}^{\prime \prime}\right) \text { if } n_{1}^{\prime}+n_{1}^{\prime \prime}-n_{2}^{\prime} \geq n_{2}\left(\text { ie } n_{1}^{\prime}+n_{1}^{\prime \prime} \geq n_{2}+n_{2}^{\prime}\right) \\
\left(n_{1}, n_{2}+n_{2}^{\prime \prime}-n_{1}^{\prime}-n_{1}^{\prime \prime}+n_{2}^{\prime}\right) \text { if } n_{1}^{\prime}+n_{1}^{\prime \prime}-n_{2}^{\prime}<n_{2}
\end{array}\right.
$$

Proof (of Lemma 4). The proof goes by induction over $k$. It is obvious for $k=1$. Asssuming it true for $k \geq 1$, we consider $k+1$. By induction hypothesis, $\mathrm{O}\left(u^{k}\right)$ is equal to $(\operatorname{Or}(u),(\operatorname{Oc}(u)-\operatorname{Or}(u)) *(k-1)+\operatorname{Oc}(u))$ if $\operatorname{Oc}(u) \geq \operatorname{Or}(u)$ and to $(\operatorname{Or}(u)+$ $(\operatorname{Or}(u)-\operatorname{Oc}(u)) *(k-1), \operatorname{Oc}(u))$ otherwise.

In the first case, $(\operatorname{Or}(u), \operatorname{Oc}(u)) \oplus(\operatorname{Or}(u),(\operatorname{Oc}(u)-\operatorname{Or}(u)) *(k-1)+\operatorname{Oc}(u))=$ $(\operatorname{Or}(u),(\operatorname{Oc}(u)-\operatorname{Or}(u))+(\operatorname{Oc}(u)-\operatorname{Or}(u)) *(k-1)+\operatorname{Oc}(u))$ as $\operatorname{Oc}(u) \geq \operatorname{Or}(u)$. In the second case, $(\operatorname{Or}(u), \operatorname{Oc}(u)) \oplus(\operatorname{Or}(u)+(\operatorname{Or}(u)-\operatorname{Oc}(u)) *(k-1), \operatorname{Oc}(u))$ is equal to $(\operatorname{Or}(u)+(\operatorname{Or}(u)-\operatorname{Oc}(u)) *(k-1)+(\operatorname{Or}(u)-\operatorname{Oc}(u)), \operatorname{Oc}(u))$ as $\operatorname{Or}(u)>\operatorname{Oc}(u)$ and thus, $\operatorname{Or}(u)+(\operatorname{Or}(u)-\operatorname{Oc}(u)) *(k-1)>\operatorname{Oc}(u)$. In both cases, the lemma holds. 


\section{B Proof of Section 3}

Proof (of Proposition 3). The non-strict inclusions are straightforward. The strict inclusions gwnVPT $\subsetneq$ awnVPT and $\mathcal{G}_{\mathrm{wn}} \subsetneq \mathcal{A}_{\mathrm{wn}}$ follow from the constraint on the range. The strict inclusion IwnVPT $\subsetneq$ gwnVPT is witnessed by $A_{2}$, as explained above.

We prove now the strict inclusion $\mathcal{L}_{\mathrm{wn}} \subsetneq \mathcal{G}_{\mathrm{wn}}$, and therefore consider the transducer $A_{1}$. Observe that $\llbracket A_{1} \rrbracket \in \mathcal{G}_{\mathrm{wn}}$, we show that $\llbracket A_{1} \rrbracket \notin \mathcal{L}_{\mathrm{wn}}$. First note that $\llbracket A_{1} \rrbracket=$ $\left\{\left(c c^{k} i r^{k} r, c c c(c r)^{k} r r(c r)^{k} r\right) \mid k \in \mathbb{N}\right\}$ and that

(Fact 1) the transduction defined by $A_{1}$ is injective

(Fact 2) any word of the output can be decomposed as $w_{1} r r w_{2}$ where $w_{1}=c c c(c r)^{k}$ and $w_{2}=(c r)^{k} r$ for some natural $k$ and for each $w_{1}$ with fixed $k$ there exists a unique $w_{2}$ such that $w_{1} r r w_{2}$ is in the range of $A_{1}$ (and conversely).

By contradiction, suppose that there exists $A_{1}^{\prime} \in$ IwnVPT such that $\llbracket A_{1}^{\prime} \rrbracket=\llbracket A_{1} \rrbracket$.

Now, for $k$ sufficiently large and depending only on the fixed size of $A_{1}^{\prime}, A_{1}^{\prime}$ has an accepting run for the input $c c^{k} i r^{k} r$ of the form given in the point $(i i)$ of Lemma 2. Let us denote by $u_{i}$ (resp. $v_{i}$ ), $i \in\{1, \ldots, 5\}$ the corresponding decomposition of the input (resp. output) word. Due to Proposition 2, words $v_{1} v_{5}, v_{2} v_{4}$ and $v_{3}$ are well-nested.

Now, assume that $v_{2}=\epsilon$ and $v_{4}=\epsilon$. Then, using a simple pumping argument over the pair $\left(u_{2}, u_{4}\right)$, one would obtain a different input producing the same output, contradicting (Fact 1 ) as $\llbracket A_{1} \rrbracket=\llbracket A_{1}^{\prime} \rrbracket$. So, $v_{2} \neq \epsilon$ or $v_{4} \neq \epsilon$.

Our aim is to identify in which $v_{j}$ 's the pattern $r r$ mentionned previously occurs.

Now, by case inspection :

- the pattern $r r$ is in $v_{1}: w_{2}$ as for suffix $v_{2} v_{3} v_{4} v_{5}$. By a pumping argument over the pair $\left(u_{2}, u_{4}\right)$ using the fact that $v_{2} \neq \epsilon$ or $v_{4} \neq \epsilon$, one would obtain a different $w_{2}^{\prime}$ such that $w_{1} r r w_{2}^{\prime}$ is in the range of $T_{2}$ (and thus $T_{1}$ ). Contradiction.

- the pattern $r r$ is in $v_{5}$ or split as the last letter of $v_{1}$ and the first letter of $v_{2}$ (resp. as the last letter of $v_{4}$ and the first letter of $v_{5}$ ): follow the lines of the previous case.

- the pattern $r r$ is in $v_{2}$ or $v_{4}$ : note that for any output the number of occurrences of the subword $r r$ is upper-bounded by 3 . Using a pumping over the pair $\left(u_{2}, u_{4}\right)$, one could obtain outputs with unboundedly many subwords $r r$. Contradiction.

- the pattern $r r$ is split as the last letter of $v_{2}$ and the first letter of $v_{3}: v_{3}$ is wellnested. Hence, it can not start with some return symbol. Contradiction.

- the pattern $r r$ is split as the last letter of $v_{3}$ and the first letter of $v_{4}$ : note that no output contains the subword $c c c c c$. As $v_{3}$ is well-nested,it must be of the form $c(c r)^{k} r$. So, $v_{1} v_{2}=c c$. If $v_{2} \neq \epsilon, v_{2}$ is equal to $c$ or $c c$. Using a pumping over the pair $\left(u_{2}, u_{4}\right)$, one could obtain outputs containing the subword ccccc. Contradiction. Otherwise, if $v_{2}=\epsilon$, then by a pumping argument over the pair $\left(u_{2}, u_{4}\right)$ using the fact that $v_{4} \neq \epsilon$, one would obtain a different $w_{2}^{\prime}$ such that $w_{1} r r w_{2}^{\prime}$ is in the range of $T_{2}$ (and thus $T_{1}$ ). Contradiction.

- the pattern $r r$ is in $v_{3}: v_{3}$ is well-nested and contains as a suffix after this pattern $r r$ a prefix of $w_{2}$ : depending on the form of this prefix:

- the prefix is of the form $(c r)^{k^{\prime}}: v_{3}$ must be of the form $c c(c r)^{k^{\prime}} r r(c r)^{k^{\prime}}$ and thus, $k^{\prime}=k$. So, $v_{1} v_{2}=c$. We then conclude as in the previous case.

- the prefix is of the form $(c r)^{k^{\prime}} c$ : this is not possible as $v_{3} \in \Sigma_{\mathrm{wn}}^{*}$. 
- the prefix is of the form $(c r)^{k} r: v_{3}$ must be of the form $c c c(c r)^{k^{\prime}} r r(c r)^{k^{\prime}}$. It implies that $v_{2}=\epsilon$ and $v_{4}=\epsilon$. Contradiction.

Hence, there is no situation in which $r r$ can occur maintaining $A_{1}^{\prime}$ to be locally wellnested. Therefore, such a $A_{1}^{\prime}$ can not exist.

\section{Proofs of Section 4}

Lemma 6. For any finite sequence of words $v_{1}, v_{2}, \ldots v_{n}$,

$$
\operatorname{Or}\left(v_{1} v_{2} \ldots v_{n}\right)=\max _{1 \leq i \leq n}\left(\operatorname{Or}\left(v_{i}\right)-\mathrm{B}\left(v_{1} v_{2} \ldots v_{i-1}\right)\right)
$$

Proof. The proof goes by induction over the length $n$ of the sequence. This is obvious for $n=1$. Now, for $n+1$ assuming this holds for $n$. $\operatorname{Or}\left(v_{1} v_{2} \ldots v_{n+1}\right)$ is equal by definition of $\oplus$ to

$$
\begin{cases}\operatorname{Or}\left(v_{1}\right) & \text { if } \left.\operatorname{Oc}\left(v_{1}\right) \geq \operatorname{Or}\left(v_{2} \ldots v_{n+1}\right)\right) \\ \operatorname{Or}\left(v_{1}\right)+\operatorname{Or}\left(v_{2} \ldots v_{n+1}\right)-\operatorname{Oc}\left(v_{2} \ldots v_{n+1}\right) & \text { if } \left.\operatorname{Oc}\left(v_{1}\right)<\operatorname{Or}\left(v_{2} \ldots v_{n+1}\right)\right)\end{cases}
$$

Now,

$$
\begin{aligned}
\operatorname{Or}\left(v_{1} v_{2} \ldots v_{n+1}\right) & =\max \left(\operatorname{Or}\left(v_{1}\right), \max _{2 \leq i \leq n+1}\left(\operatorname{Or}\left(v_{i}\right)-\mathrm{B}\left(v_{1} v_{2} \ldots v_{i-1}\right)\right)\right) \\
& =\max \left(\operatorname{Or}\left(v_{1}\right), \max _{2 \leq i \leq n+1}\left(\operatorname{Or}\left(v_{i}\right)-\mathrm{B}\left(v_{2} \ldots v_{i-1}\right)\right)-\mathrm{B}\left(v_{1}\right)\right) \\
& \left.=\max \left(\operatorname{Or}\left(v_{1}\right), \operatorname{Or}\left(v_{2} \ldots v_{n+1}\right)-\mathrm{B}\left(v_{1}\right)\right) \quad \text { (by ind. hyp. }\right)
\end{aligned}
$$

Now depending of the greatest of the two elements; if $\operatorname{Or}\left(v_{1}\right) \geq \operatorname{Or}\left(v_{2} \ldots v_{n+1}\right)-$ $\mathrm{B}\left(v_{1}\right)=\operatorname{Or}\left(v_{2} \ldots v_{n+1}\right)-\operatorname{Oc}\left(v_{1}\right)+\operatorname{Or}\left(v_{1}\right)$. We have $\operatorname{Oc}\left(v_{1}\right) \geq \operatorname{Or}\left(v_{2} \ldots v_{n+1}\right)$. If $\operatorname{Or}\left(v_{1}\right)<\operatorname{Or}\left(v_{2} \ldots v_{n+1}\right)-\mathrm{B}\left(v_{1}\right)=\operatorname{Or}\left(v_{2} \ldots v_{n+1}\right)-\operatorname{Oc}\left(v_{1}\right)+\operatorname{Or}\left(v_{1}\right)$. Then, $\operatorname{Oc}\left(v_{1}\right)<\operatorname{Or}\left(v_{2} \ldots v_{n+1}\right)$. In both cases, this is equal to $\operatorname{Or}\left(v_{1} v_{2} \ldots v_{n+1}\right)$.

Proof (of Lemma 5). Let $A=(Q, I, F, \Gamma, \delta)$ and $\rho$ be an accepting run such that $|\rho| \geq$ $N_{A}$. We distinguish two cases, depending on height $(\rho)$ :

- when height $(\rho)<2|Q|^{2}$ : in this case, by definition of $N_{A}$, we can apply Lemma 2.(i) twice and prove that $\rho$ is of the following form:

$$
(i, \perp) \stackrel{u_{1} / v_{1}}{\longrightarrow}(p, \sigma) \stackrel{u_{2} / v_{2}}{\longrightarrow}(p, \sigma) \stackrel{u_{3} / v_{3}}{\longrightarrow}\left(q, \sigma^{\prime}\right) \stackrel{u_{4} / v_{4}}{\longrightarrow}\left(q, \sigma^{\prime}\right) \stackrel{u_{5} / v_{5}}{\longrightarrow}(f, \perp)
$$

with $u_{2}, u_{4} \in \Sigma_{\mathrm{wn}}^{*} \backslash\{\epsilon\}$. Then, by criterion $(C 1)$, we have $\mathrm{B}\left(v_{2}\right)=\mathrm{B}\left(v_{4}\right)=0$. One can prove that at least one of $u_{2}$ and $u_{4}$ can be removed from $u$ while preserving the value $\operatorname{Or}(u)$. Let us denote by $v^{\prime}$ the resulting output word. Observe also that removing this part of the run does not modify the balance $\mathrm{B}($.$) of the run, as$ $\mathrm{B}\left(v_{2}\right)=\mathrm{B}\left(v_{4}\right)=0$. As $\mathrm{Oc}(v)=\mathrm{B}(v)+\operatorname{Or}(v)$, we obtain $\mathrm{O}(v)=\mathrm{O}\left(v^{\prime}\right)$, yielding the result.

- when height $(\rho) \geq 2|Q|^{2}$ : in this case, we can apply Lemma 2.(ii) twice and prove that $\rho$ is of the following form:

$$
(i, \perp) \stackrel{u_{1} / v_{1}}{\longrightarrow}\left(p_{1}, \sigma\right) \stackrel{u_{2} / v_{2}}{\longrightarrow}\left(p_{1}, \sigma \sigma_{1}\right) \stackrel{u_{3} / v_{3}}{\longrightarrow}\left(q_{1}, \sigma \sigma_{1} \sigma_{2}\right) \stackrel{u_{4} / v_{4}}{\longrightarrow}\left(q_{1}, \sigma \sigma_{1} \sigma_{2} \sigma_{3}\right)
$$


$\stackrel{u_{5} / v_{5}}{\longrightarrow}\left(q_{2}, \sigma \sigma_{1} \sigma_{2} \sigma_{3}\right) \stackrel{u_{6} / v_{6}}{\longrightarrow}\left(q_{2}, \sigma \sigma_{1} \sigma_{2}\right) \stackrel{u_{7} / v_{8}}{\longrightarrow}\left(p_{2}, \sigma \sigma_{1}\right) \stackrel{u_{8} / v_{8}}{\longrightarrow}\left(p_{2}, \sigma\right) \stackrel{u_{9} / v_{9}}{\longrightarrow}$

$(f, \perp)$, with $u_{1} u_{9}, u_{2} u_{8}, u_{3} u_{7}, u_{4} u_{6}, u_{5} \in \Sigma_{\text {wn }}^{*}$ and $\sigma_{1}, \sigma_{3} \neq \perp$.

Then the two following runs can be built: the one obtained by removing the parts of $\rho$ on $u_{2}$ and $u_{8}$, and the one obtained by removing the parts of $\rho$ on $u_{4}$ and $u_{6}$, yielding runs whose length is strictly smaller than $|\rho|$. Let us denote these two runs by $\rho^{\prime}$ and $\rho^{\prime \prime}$ respectively, and their outputs by $v^{\prime}$ and $v^{\prime \prime}$. As $A$ verifies the criterion $(C 2)$, we have that $\mathrm{B}(v)=\mathrm{B}\left(v^{\prime}\right)=\mathrm{B}\left(v^{\prime \prime}\right)$, as $\mathrm{B}\left(v_{2}\right)+\mathrm{B}\left(v_{8}\right)=\mathrm{B}\left(v_{4}\right)+\mathrm{B}\left(v_{6}\right)=0$ and $\mathrm{B}$ is commutative. In order to obtain the result, we study $\operatorname{Or}(v)$.

By Lemma 6,

$$
\operatorname{Or}(v)=\max _{i=1}^{9}\left\{\operatorname{Or}\left(v_{i}\right)-\mathrm{B}\left(v_{1} \ldots v_{i-1}\right)\right\}
$$

Then, we distinguish two cases:

- If the maximum corresponding to $\operatorname{Or}(v)$ is not obtained for $i \in\{4,5,6\}$. In this case, we will prove that $\operatorname{Or}\left(v^{\prime \prime}\right) \geq \operatorname{Or}(v)$. When we remove parts of $\rho$ on $u_{4}$ and $u_{6}$, we have:

$$
\begin{aligned}
& \operatorname{Or}\left(v^{\prime \prime}\right)=\max \left\{\operatorname{Or}\left(v_{1}\right), \operatorname{Or}\left(v_{2}\right)-\mathrm{B}\left(v_{1}\right), \operatorname{Or}\left(v_{3}\right)-\mathrm{B}\left(v_{1} v_{2}\right), \operatorname{Or}\left(v_{5}\right)-\mathrm{B}\left(v_{1} v_{2} v_{3}\right),\right. \\
& \left.\operatorname{Or}\left(v_{7}\right)-\mathrm{B}\left(v_{1} v_{2} v_{3} v_{5}\right), \operatorname{Or}\left(v_{8}\right)-\mathrm{B}\left(v_{1} v_{2} v_{3} v_{5} v_{7}\right), \operatorname{Or}\left(v_{9}\right)-\mathrm{B}\left(v_{1} v_{2} v_{3} v_{5} v_{7} v_{8}\right)\right\}
\end{aligned}
$$

As $\mathrm{B}\left(v_{4}\right)+\mathrm{B}\left(v_{6}\right)=0$ and the maximum corresponding to $\operatorname{Or}(v)$ is not obtained for $i \in\{4,5,6\}$, we obtain:

$$
\operatorname{Or}\left(v^{\prime \prime}\right)=\max \left\{\operatorname{Or}(v), \operatorname{Or}\left(v_{5}\right)-\mathrm{B}\left(v_{1} v_{2} v_{3}\right)\right\}
$$

This proves $\operatorname{Or}\left(v^{\prime \prime}\right) \geq \operatorname{Or}(v)$. As for any word $w$ we have $\operatorname{Oc}(w)=\mathrm{B}(w)+$ $\operatorname{Or}(w)$ we also have $\operatorname{Oc}\left(v^{\prime \prime}\right) \geq \operatorname{Oc}(v)$. This entails $\left\|\mathrm{O}\left(v^{\prime \prime}\right)\right\| \geq\|\mathrm{O}(v)\|$.

- If the maximum corresponding to $\operatorname{Or}(v)$ is obtained for $i \in\{4,5,6\}$. In this case, we will prove that $\operatorname{Or}\left(v^{\prime}\right) \geq \operatorname{Or}(v)$. When we remove parts of $\rho$ on $u_{2}$ and $u_{8}$, denoting by $v^{\prime}$ the resulting output word, we have:

$$
\begin{aligned}
& \operatorname{Or}\left(v^{\prime}\right)=\max \left\{\operatorname{Or}\left(v_{1}\right), \operatorname{Or}\left(v_{3}\right)-\mathrm{B}\left(v_{1}\right), \operatorname{Or}\left(v_{4}\right)-\mathrm{B}\left(v_{1} v_{3}\right), \operatorname{Or}\left(v_{5}\right)-\mathrm{B}\left(v_{1} v_{3} v_{4}\right),\right. \\
& \left.\quad \operatorname{Or}\left(v_{6}\right)-\mathrm{B}\left(v_{1} v_{3} v_{4} v_{5}\right), \operatorname{Or}\left(v_{7}\right)-\mathrm{B}\left(v_{1} v_{3} v_{4} v_{5} v_{6}\right), \operatorname{Or}\left(v_{9}\right)-\mathrm{B}\left(v_{1} v_{3} v_{4} v_{5} v_{6} v_{7}\right)\right\}
\end{aligned}
$$

As the maximum corresponding to $\operatorname{Or}(v)$ is obtained for $i \in\{4,5,6\}$, we can write:

$$
\begin{aligned}
\operatorname{Or}(v) & =\max \left\{\operatorname{Or}\left(v_{4}\right)-\mathrm{B}\left(v_{1} v_{2} v_{3}\right), \operatorname{Or}\left(v_{5}\right)-\mathrm{B}\left(v_{1} v_{2} v_{3} v_{4}\right), \operatorname{Or}\left(v_{6}\right)-\mathrm{B}\left(v_{1} v_{2} v_{3} v_{4} v_{5}\right)\right\} \\
& =\max \left\{\operatorname{Or}\left(v_{4}\right)-\mathrm{B}\left(v_{1} v_{3}\right), \operatorname{Or}\left(v_{5}\right)-\mathrm{B}\left(v_{1} v_{3} v_{4}\right), \operatorname{Or}\left(v_{6}\right)-\mathrm{B}\left(v_{1} v_{3} v_{4} v_{5}\right)\right\}-\mathrm{B}\left(v_{2}\right)
\end{aligned}
$$

By criterion $(C 2)$, we have $\mathrm{B}\left(v_{2}\right) \geq 0$ and thus:

$$
\max \left\{\operatorname{Or}\left(v_{4}\right)-\mathrm{B}\left(v_{1} v_{3}\right), \operatorname{Or}\left(v_{5}\right)-\mathrm{B}\left(v_{1} v_{3} v_{4}\right), \operatorname{Or}\left(v_{6}\right)-\mathrm{B}\left(v_{1} v_{3} v_{4} v_{5}\right)\right\} \geq \operatorname{Or}(v)
$$

In addition, by Lemma 6 , we have $\operatorname{Or}(v) \geq \operatorname{Or}\left(v_{1}\right)$ and $\operatorname{Or}(v) \geq \operatorname{Or}\left(v_{9}\right)-$ $\mathrm{B}\left(v_{1} v_{2} v_{3} v_{4} v_{5} v_{6} v_{7} v_{8}\right)$. By criterion $(C 2)$, the latter property can be written as $\operatorname{Or}(v) \geq \operatorname{Or}\left(v_{9}\right)-\mathrm{B}\left(v_{1} v_{3} v_{4} v_{5} v_{6} v_{7}\right)$ thanks to the property $\mathrm{B}\left(v_{2}\right)+\mathrm{B}\left(v_{8}\right)=0$. Using these facts, we can simplify the previous expression and obtain:

$$
\begin{aligned}
\operatorname{Or}\left(v^{\prime}\right)= & \max \left\{\operatorname{Or}\left(v_{3}\right)-\mathrm{B}\left(v_{1}\right), \operatorname{Or}\left(v_{4}\right)-\mathrm{B}\left(v_{1} v_{3}\right), \operatorname{Or}\left(v_{5}\right)-\mathrm{B}\left(v_{1} v_{3} v_{4}\right),\right. \\
& \left.\operatorname{Or}\left(v_{6}\right)-\mathrm{B}\left(v_{1} v_{3} v_{4} v_{5}\right), \operatorname{Or}\left(v_{7}\right)-\mathrm{B}\left(v_{1} v_{3} v_{4} v_{5} v_{6}\right)\right\} \\
= & \max \left\{\operatorname{Or}\left(v_{3}\right)-\mathrm{B}\left(v_{1} v_{2}\right), \operatorname{Or}\left(v_{4}\right)-\mathrm{B}\left(v_{1} v_{2} v_{3}\right), \operatorname{Or}\left(v_{5}\right)-\mathrm{B}\left(v_{1} v_{2} v_{3} v_{4}\right),\right. \\
& \left.\operatorname{Or}\left(v_{6}\right)-\mathrm{B}\left(v_{1} v_{2} v_{3} v_{4} v_{5}\right), \operatorname{Or}\left(v_{7}\right)-\mathrm{B}\left(v_{1} v_{2} v_{3} v_{4} v_{5} v_{6}\right)\right\}+\mathrm{B}\left(v_{2}\right) \\
= & \operatorname{Or}(v)+\mathrm{B}\left(v_{2}\right)
\end{aligned}
$$


The last equality follows from the fact the maximum corresponding to $\operatorname{Or}(v)$ is obtained for $i \in\{4,5,6\}$. This concludes this case.

\section{Proofs of Section 5}

Proof (of Proposition 6). We consider only the third point which combined the first two. Is is well-know that the set of stacks of a pushdown automata obtained along successful computations is a regular language. For a VPT $A$, it is easy to built in polynomial time a finite state automaton $A_{p}^{\text {reach }}$ (resp. $A_{p}^{\text {coreach }}$ ) over $\Gamma$ such a word $\omega$ is accepted by $A_{p}^{\text {reach }}$ iff $(p, \omega)$ is reachable (resp. co-reachable) in $A$. It is then enough to consider pairs $\left(p, p^{\prime}\right)$ such that $L\left(A_{p}^{\text {reach }}\right) \cap L\left(A_{p^{\prime}}^{\text {coreach }}\right) \neq \emptyset$.

Proof (of Theorem 3). By Theorem 1, deciding the class awnVPT amounts to decide criteria $(C 1)$ and $(C 2)$. Therefore we propose a non-deterministic algorithm running in polynomial space, yielding the result thanks to Savitch theorem.

We claim that $A$ verifies $(C 1)$ and $(C 2)$ if and only if it verifies these criteria on "small instances", defined as follows:

- Criterion $(C 1)$ : consider only words $u_{2}$ such that height $\left(u_{2}\right) \leq|Q|^{2}$ and $\left|u_{2}\right| \leq$ 2. $|Q|^{|Q|^{2}}$.

- Criterion $(C 2)$ : consider only stacks $\sigma^{\prime}$ such that $\left|\sigma^{\prime}\right| \leq|Q|^{2}$ and words $u_{2}, u_{4}$ of height at most $2 .|Q|^{2}$ and length at most $|Q|^{2} \cdot|Q|^{|Q|^{2}}$.

The non-deterministic algorithm follows from the claim: in order to exhibit a witness of the fact that $A \notin$ awnVPT, the algorithm guesses whether $(C 1)$ or $(C 2)$ is violated, and a pair of states $(p, q)$ and on the fly, one or two runs, according to the criterion, of exponential size, which can be done in polynomial space (using counters keeping track of the current length of the guessed run(s)). Using Proposition 6 it also verifies that there exists a stack $\sigma$ such that $(p, \sigma)$ is reachable and $(q, \sigma)$ is co-reachable.

To prove this claim, we will show, by induction on $u \in \Sigma_{\mathrm{wn}}^{*}$, that for every run $\rho:(p, \perp) \stackrel{u \mid v}{\longrightarrow}(q, \perp)$ that can be completed into an accepting run, and for every decomposition of this run according to criterion $\left(C_{1}\right)$ or $(C 2)$, the property stated by the corresponding criterion is fulfilled. Formally, this means that:

- for every decomposition $\rho:(p, \perp) \stackrel{u_{1} / v_{1}}{\longrightarrow}\left(p^{\prime}, \sigma\right) \stackrel{u_{2} / v_{2}}{\longrightarrow}\left(p^{\prime}, \sigma\right) \stackrel{u_{3} / v_{3}}{\longrightarrow}(q, \perp)$ with $u_{1} u_{3}, u_{2} \in \Sigma_{\mathrm{wn}}^{*}$, we have $\mathrm{B}\left(v_{2}\right)=0$,

- for every decomposition $\rho:(p, \perp) \stackrel{u_{1} / v_{1}}{\longrightarrow}\left(p^{\prime}, \sigma\right) \stackrel{u_{2} / v_{2}}{\longrightarrow}\left(p^{\prime}, \sigma \sigma^{\prime}\right) \stackrel{u_{3} / v_{3}}{\longrightarrow}\left(q^{\prime}, \sigma \sigma^{\prime}\right) \stackrel{u_{4} / v_{4}}{\longrightarrow}$ $\left(q^{\prime}, \sigma\right) \stackrel{u_{5} / v_{5}}{\longrightarrow}(q, \perp)$ with $u_{2} u_{4}, u_{3} \in \Sigma_{\mathrm{wn}}^{*}$ and $\sigma^{\prime} \neq \perp$, we have $\mathrm{B}\left(v_{2}\right)+\mathrm{B}\left(v_{4}\right)=0$ and $\mathrm{B}\left(v_{2}\right) \geq 0$.

We now suppose that criteria $(C 1)$ and $(C 2)$ are verified on small instances, as defined above, and prove, by induction on $u \in \Sigma_{\mathrm{wn}}^{*}$, that the VPT $A$ verifies the criteria $(C 1)$ and $(C 2)$ on every decomposition of a run on $u$.

Cases $u=\epsilon$ and $u=a \in \Sigma_{i}$ : The result directly follows from the hypothesis as they are small words. 
Case $u=u_{1} u_{2}$ with $u_{1}, u_{2} \neq \epsilon$. Consider a run $\rho:(p, \perp) \stackrel{u_{1} u_{2} \mid v}{\longrightarrow}(q, \perp)$. First, consider a decomposition of $\rho$ according to criterion $(C 2)$. This decomposition is necessarily either completely in the sub-run on $u_{1}$, or in that on $u_{2}$. The result follows by induction. Second, consider a decomposition of $\rho$ according to criterion $(C 1)$. If the decomposition is completely in the sub-run on $u_{1}$, or in that on $u_{2}$, then the result follows by induction. Otherwise, the decomposition of $\rho$ looks as follows: $\rho$ : $(p, \perp) \stackrel{u_{1}^{\prime} \mid v_{1}^{\prime}}{\longrightarrow}\left(p_{1}, \perp\right) \stackrel{u_{1}^{\prime \prime} \mid v_{1}^{\prime \prime}}{\longrightarrow}\left(p_{2}, \perp\right) \stackrel{u_{2}^{\prime} \mid v_{2}^{\prime}}{\longrightarrow}\left(p_{1}, \perp\right) \stackrel{u_{2}^{\prime \prime} \mid v_{2}^{\prime \prime}}{\longrightarrow}(q, \perp)$ where $u_{i}=u_{i}^{\prime} u_{i}^{\prime \prime}$ for $i \in\{1,2\}$. Let us denote by $\rho_{1}$ the run $\left(p_{1}, \perp\right) \stackrel{u_{1}^{\prime \prime} \mid v_{1}^{\prime \prime}}{\longrightarrow}\left(p_{2}, \perp\right)$ and by $\rho_{2}$ the run $\left(p_{2}, \perp\right) \stackrel{u_{2}^{\prime} \mid v_{2}^{\prime}}{\longrightarrow}\left(p_{1}, \perp\right)$. The loop under consideration is represented by the run $\rho_{1} \rho_{2}$.

By induction hypothesis applied on $u_{1}$, any decomposition of $\rho_{1}$ according to criteria $(C 1)$ and $(C 2)$ fulfills these criteria. Using Lemma 2, one can identify such decompositions if the height or the length of the run is large enough. Using the according criterion and the definition of the mapping $\mathrm{B}($.$) , one can remove the identified loop$ while preserving the value of $\mathrm{B}($.$) . Applying iteratively this process, we can build from$ $\rho_{1}$ a run $\rho_{1}^{\prime}$ such that $\mathrm{B}\left(\rho_{1}^{\prime}\right)=\mathrm{B}\left(\rho_{1}\right)$, height $\left(\rho_{1}^{\prime}\right)<|Q|^{2}$ and $\left|\rho_{1}^{\prime}\right|<|Q|^{|Q|^{2}}$.

A similar construction can be done for $\rho_{2}$, yielding some run $\rho_{2}^{\prime}$.

By construction of $\rho_{1}^{\prime}$ and $\rho_{2}^{\prime}$, we have $\mathrm{B}\left(\rho_{1}^{\prime} \rho_{2}^{\prime}\right)=\mathrm{B}\left(\rho_{1} \rho_{2}\right)$. Moreover, it is routine to verify that the run $\rho_{1}^{\prime} \rho_{2}^{\prime}$ verifies the constraints of the hypothesis on its height and length, and thus $\mathrm{B}\left(\rho_{1}^{\prime} \rho_{2}^{\prime}\right)=0$.

Case cur. We consider some run $\rho$ as follows $\rho:(p, \perp) \stackrel{c \mid v_{0}}{\longrightarrow}\left(p^{\prime}, \gamma\right) \stackrel{u \mid v}{\longrightarrow}\left(q^{\prime}, \gamma\right) \stackrel{r \mid v_{4}}{\longrightarrow}$ $(q, \perp)$.

We first consider a decomposition of $\rho$ according to criterion $(C 1)$. If it is completely in the sub-run on $u$, then the result follows by induction. Otherwise, we have $p=q$ and the run $\rho$ itself verifies the conditions of criterion $(C 1)$. Using standard horizontal and vertical pumping and the induction hypothesis on $u$, we can assume that the height of $u$ is strictly less than $|Q|^{2}$ and that the length of $u$ is strictly less than $|Q|^{\left(\left.Q\right|^{2}\right.}$ (otherwise by a pumping reasoning we can exhibit a decomposition that satisfies either criterion $(C 1)$ or criterion $(C 2)$ and using the induction hypothesis remove this part of $u$ ). Then, the word cur verifies our initial requirements on the height and the size, and we thus have $\mathrm{B}\left(v_{0} v v_{4}\right)=0$, as expected.

Consider now a decomposition of $\rho$ according to criterion $(C 2)$. If it is completely in the sub-run on $u$, then the result follows by induction. Otherwise, there exists a decomposition of $u$ as $u=u_{1} u_{2} u_{3}$, with $u_{1} u_{3}, u_{2} \in \Sigma_{\mathrm{wn}}^{*}$, and there exists a run $\left(p^{\prime}, \perp\right) \stackrel{u_{1} \mid v_{1}}{\longrightarrow}(p, \sigma) \stackrel{u_{2} \mid v_{2}}{\longrightarrow}(q, \sigma) \stackrel{u_{3} \mid v_{3}}{\longrightarrow}\left(q^{\prime}, \perp\right)$. First, if $|\sigma| \geq|Q|^{2}$, then one can find in this run a decomposition according to criterion $(C 2)$, and by induction hypothesis on $u$, the corresponding matched loops have a null effect in the computation of $\mathrm{B}$. When removing these matched loops, we thus obtain new input words $u_{1}^{\prime}$ and $u_{3}^{\prime}$ with output words $v_{1}^{\prime}$ and $v_{3}^{\prime}$ such that $\mathrm{B}\left(v_{1}^{\prime}\right)+\mathrm{B}\left(v_{3}^{\prime}\right)=\mathrm{B}\left(v_{1}\right)+\mathrm{B}\left(v_{3}\right)$, and $\mathrm{B}\left(v_{1}\right) \geq \mathrm{B}\left(v_{1}^{\prime}\right)$. We thus assume now that $|\sigma|<\left|Q^{2}\right|$ and let $k=|\sigma|$.

We decompose $u_{1}^{\prime}$ as follows: $u_{1}^{\prime}=w_{0} c_{1} w_{1} c_{2} \ldots c_{k} w_{k}$. Using standard horizontal and vertical pumping (Lemma 2) and the induction hypothesis on $u$, we can assume that the height of $w_{i}$ 's is strictly less than $|Q|^{2}$ and that the length of $w_{i}$ 's is strictly less than $|Q|^{|Q|^{2}}$. A similar reasoning can be done on word $u_{3}^{\prime}$. Thus, the two words $c u_{1}^{\prime}$ and $u_{3}^{\prime} r$ 
verify the conditions on their height and size to ensure that $\mathrm{B}\left(v_{0} v_{1}^{\prime}\right)+\mathrm{B}\left(v_{3}^{\prime} v_{4}\right)=0$, and $\mathrm{B}\left(v_{0} v_{1}^{\prime}\right) \geq 0$. This entails $\mathrm{B}\left(v_{0} v_{1}\right)+\mathrm{B}\left(v_{3} v_{4}\right)=0$, and $\mathrm{B}\left(v_{0} v_{1}\right) \geq 0$ as we wanted to prove.

This concludes the induction, and thus the proof.

\section{E Proofs of Section 6}

This section is devoted to the proof of Theorem 6 .

We define the set $\mathcal{A C}$ of pairs $\left(p, p^{\prime}\right)$ such that for some $\sigma,(p, \sigma)$ is reachable and $\left(p^{\prime}, \sigma\right)$ is co-reachable. This set can be computed in polynomial time thanks to Proposition 6.

For a VPT $A$, we consider triples of the form $\left(p, q,\left(n_{1}, n_{2}\right)\right)$ where $p, q \in Q$ such that $(p, q) \in \mathcal{A C}$ and $\left(n_{1}, n_{2}\right) \in \mathbb{N} \times \mathbb{N}$. We consider the set $\mathcal{S}$ of such triples, as the least one satisfying the rules from Figure 2.

$$
\begin{gathered}
\frac{(p, p) \in \mathcal{A C}}{(p, p,(0,0)) \in \mathcal{S}}(\mathrm{R} 0) \\
\frac{\left(p^{\prime}, c, \gamma, v_{1}, p\right) \in \delta_{c},\left(q, r, \gamma, v_{2}, q^{\prime}\right) \in \delta_{r},\left(p^{\prime}, q^{\prime \prime}\right) \in \mathcal{A C}}{\left(p, q,\left(n_{1}, n_{2}\right)\right) \in \mathcal{S},\left(q^{\prime}, q^{\prime \prime},\left(n_{1}^{\prime}, n_{2}^{\prime}\right)\right) \in \mathcal{S}} \\
\frac{\left(p^{\prime}, q^{\prime \prime}, \mathrm{O}\left(v_{1}\right) \oplus\left(n_{1}, n_{2}\right) \oplus \mathrm{O}\left(v_{2}\right) \oplus\left(n_{1}^{\prime}, n_{2}^{\prime}\right)\right) \in \mathcal{S}}{(p, i, v, q) \in \delta_{i},\left(q, q^{\prime},\left(n_{1}, n_{2}\right)\right) \in \mathcal{S},\left(p, q^{\prime}\right) \in \mathcal{A C}} \\
\frac{\left(p, q^{\prime}, \mathrm{O}(v) \oplus\left(n_{1}, n_{2}\right)\right) \in \mathcal{S}}{\mathrm{S} 2}
\end{gathered}
$$

Fig. 2.

Proposition 8. For any VPT $A, \mathcal{S}=\mathcal{U P} \mathcal{S}_{A}$.

Proof. The proof goes by induction over the structure of runs for well-nested input words of the form $\epsilon, a u, c u_{1} r u_{2}$.

Proposition 9. For $A$ in awnVPT, the set $\mathcal{S}$ is finite and can be computed in exponential time.

Proof. Obviously, rules from Figure 2 can be turned into an algorithm whose iterations will inspect each inference rule for each possible inputs. Such an iteration may add at least one new triple in $\mathcal{S}$. By Proposition 8 and Proposition 5, it is known that in triples $\left(p, p^{\prime},\left(n_{1}, n_{2}\right), n_{1}, n_{2}\right.$ are bounded exponentially in the size of $A$ which ensured termination for the algorithm.

We consider the following definition:

Definition 9. Given a run $\rho:(p, \perp) \stackrel{u \mid v}{\longrightarrow}\left(p^{\prime}, \perp\right)$ of $A$, we define $\operatorname{MAX}-\operatorname{BAL}(\rho)$ as the largest non-negative integer $n$ such that $\rho$ can be decomposed as $\rho:(p, \perp) \stackrel{u_{1} \mid v_{1}}{\longrightarrow}$ $\left(p_{1}, \perp\right) \stackrel{u_{2} \mid v_{2}}{\longrightarrow}\left(p^{\prime}, \perp\right)$ and $n=\operatorname{Or}\left(v_{2}\right)$ or $n=\operatorname{Oc}\left(v_{1}\right)$. 
The following lemma states that a run of $A$ and a run of $B$ can be combined to build a run of $C$, provided there exists a word $v \in \Delta^{*}$ produced by the run of $A$, and taken as input of the run of $B$. Observe also that we may add a stack in the state of $C$, considered as an unused stack added "below" the run of $B$, provided the size of the resulting stack stored in the state never exceeds the bound $K$. This is obtained thanks to the definition of MAX-BAL.

Lemma 7. If we have:

- $\rho:(p, \perp) \stackrel{u \mid v}{\longrightarrow}\left(p^{\prime}, \perp\right)$ is a run of $A$

- $\sigma, \sigma^{\prime} \in \Gamma_{B}^{*}$ such that $\mathrm{O}(v)=\left(|\sigma|,\left|\sigma^{\prime}\right|\right)$

- $(q, \sigma) \stackrel{v \mid w}{\longrightarrow}\left(q^{\prime}, \sigma^{\prime}\right)$ is a run of $B$

Then $\left(\left(p, q, \sigma_{0} \sigma\right), \perp\right) \stackrel{u \mid w}{\longrightarrow}\left(\left(p^{\prime}, q^{\prime}, \sigma_{0} \sigma^{\prime}\right), \perp\right)$ is a run of $C$, for every stack $\sigma_{0} \in$ $\Gamma_{B}^{\leq K-\operatorname{MAX}-\operatorname{BAL}(\rho)}$

Proof. We proceed by induction on some word $u^{\prime} \in \Sigma_{\mathrm{wn}}^{*}$.

Cases $u^{\prime}=\epsilon, u^{\prime} \in \Sigma_{i}$ : these cases directly follows from the definition of $C$.

Case $u^{\prime}=u_{1} u_{2}$. We have $\rho=\rho_{1} \rho_{2}$ with $\rho_{1}:(p, \perp) \stackrel{u_{1} \mid v_{1}}{\longrightarrow}\left(p_{1}, \perp\right)$ and $\rho_{2}$ : $\left(p_{1}, \perp\right) \stackrel{u_{2} \mid v_{2}}{\longrightarrow}\left(p^{\prime}, \perp\right)$, and a run $(q, \sigma) \stackrel{v_{1} \mid w_{1}}{\longrightarrow}\left(q_{1}, \sigma_{1}\right) \stackrel{v_{2}, w_{2}}{\longrightarrow}\left(q^{\prime}, \sigma^{\prime}\right)$ of $B$.

We let $\mathrm{O}(v)=(n, m), \mathrm{O}\left(v_{1}\right)=\left(n_{1}, m_{1}\right)$ and $\mathrm{O}\left(v_{2}\right)=\left(n_{2}, m_{2}\right)$. We have $(n, m)=\left(n_{1}, m_{1}\right) \oplus\left(n_{2}, m_{2}\right)$.

There exist two stacks $\tilde{\sigma_{\perp}}, \overline{\sigma_{\perp}} \in \Gamma_{B}^{*}$ such that:

1. $\sigma=\tilde{\sigma_{\perp}} \tilde{\sigma}$ and $|\tilde{\sigma}|=n_{1}$

2. $\sigma_{1}=\tilde{\sigma_{\perp}} \tilde{\sigma_{1}}$ and $|\tilde{\sigma}|=m_{1}$

3. $\sigma_{1}=\overline{\sigma_{\perp} \sigma_{1}}$ and $\left|\overline{\sigma_{1}}\right|=n_{2}$

4. $\sigma^{\prime}=\overline{\sigma_{\perp}} \sigma^{\prime}$ and $\left|\bar{\sigma}^{\prime}\right|=m_{2}$

One of $\tilde{\sigma_{\perp}}$ and $\overline{\sigma_{\perp}}$ is actually the empty stack.

Thanks to $\mathrm{O}\left(v_{1}\right)=\left(n_{1}, m_{1}\right)$ and $\mathrm{O}\left(v_{2}\right)=\left(n_{2}, m_{2}\right)$, the following runs exist in $B$ :

- $(q, \tilde{\sigma}) \stackrel{v_{1} \mid w_{1}}{\longrightarrow}\left(q_{1}, \tilde{\sigma_{1}}\right)$

- $\left(q_{1}, \overline{\sigma_{1}}\right) \stackrel{v_{2}, w_{2}}{\longrightarrow}\left(q^{\prime}, \bar{\sigma}^{\prime}\right)$

Let $\sigma_{0} \in \Gamma_{\bar{B}}^{\leq K-\operatorname{MAX}-\operatorname{BaL}(\rho)}$. We can easily prove that $\operatorname{MAX}-\operatorname{BAL}(\rho)=\max \left(\operatorname{MAX}-\operatorname{BAL}\left(\rho_{1}\right)+\right.$ $\left.\left|\tilde{\sigma_{\perp}}\right|, \operatorname{MAX}-\operatorname{BAL}\left(\rho_{2}\right)+\left|\overline{\sigma_{\perp}}\right|\right)$. We thus have $\left|\sigma_{0} \tilde{\sigma_{\perp}}\right| \leq K-\operatorname{MAX}-\operatorname{BAL}\left(\rho_{1}\right)$ and $\left|\sigma_{0} \overline{\sigma_{\perp}}\right| \leq$ $K-\operatorname{MAX}-\operatorname{BAL}\left(\rho_{2}\right)$. By induction hypothesis applied on $u_{1}$ and $u_{2}$, we obtain:

- $\left(\left(p, q, \sigma_{0} \tilde{\sigma_{\perp}} \tilde{\sigma}\right), \perp\right) \stackrel{u_{1} \mid w_{1}}{\longrightarrow}\left(\left(p_{1}, q_{1}, \sigma_{0} \tilde{\sigma_{\perp}} \tilde{\sigma_{1}}\right), \perp\right)$ is a run of $C$.

- $\left(\left(p_{1}, q_{1}, \sigma_{0} \overline{\sigma_{\perp} \sigma_{1}}\right), \perp\right) \stackrel{u_{2} \mid w_{2}}{\longrightarrow}\left(\left(p^{\prime}, q^{\prime}, \sigma_{0} \overline{\sigma_{\perp}} \bar{\sigma}^{\prime}\right), \perp\right)$ is a run of $C$.

This concludes the proof for this case.

Case $u^{\prime}=$ cur: We can decompose the run $\rho$ in $A$ on cur as follows:

$$
\rho:(p, \perp) \stackrel{c \mid v_{1}}{\longrightarrow}\left(p_{1}, \gamma\right) \stackrel{u \mid v_{2}}{\longrightarrow}\left(p_{1}^{\prime}, \gamma\right) \stackrel{r \mid v_{3}}{\longrightarrow}\left(p^{\prime}, \perp\right)
$$


Let $v=v_{1} v_{2} v_{3}$, we write $\mathrm{O}(v)=(n, m)$, and $\mathrm{O}\left(v_{i}\right)=\left(n_{i}, m_{i}\right)$ for $i=1,2,3$.

We can decompose the run in $B$ on the word $v$ as follows:

$$
(q, \sigma) \stackrel{v_{1} \mid w_{1}}{\longrightarrow}\left(q_{1}, \sigma_{1}\right) \stackrel{v_{2} \mid w_{2}}{\longrightarrow}\left(q_{2}, \sigma_{2}\right) \stackrel{v_{3} \mid w_{3}}{\longrightarrow}\left(q^{\prime}, \sigma^{\prime}\right)
$$

with $\mathrm{O}(v)=\left(|\sigma|,\left|\sigma^{\prime}\right|\right)$.

There exist stacks $\sigma_{\perp}, \overline{\sigma_{\perp}}, \hat{\sigma_{\perp}}$ such that:

1. $\sigma=\sigma_{\perp} \tilde{\sigma}$ and $|\tilde{\sigma}|=n_{1}$

2. $\sigma_{1}=\sigma_{\perp} \tilde{\sigma_{1}}$ and $\left|\tilde{\sigma_{1}}\right|=m_{1}$

3. $\sigma_{1}=\overline{\sigma_{\perp} \sigma_{1}}$ and $\left|\bar{\sigma}_{1}\right|=n_{2}$

4. $\sigma_{2}=\overline{\sigma_{\perp} \sigma_{2}}$ and $\left|\overline{\sigma_{2}}\right|=m_{2}$

5. $\sigma_{2}=\hat{\sigma_{\perp}} \hat{\sigma_{2}}$ and $\left|\hat{\sigma_{2}}\right|=n_{3}$

6. $\sigma^{\prime}=\hat{\sigma_{\perp}} \hat{\sigma}^{\prime}$ and $\left|\hat{\sigma}^{\prime}\right|=m_{3}$

By points 1 and 2, we have: $(q, \tilde{\sigma}) \stackrel{v_{1} \mid w_{1}}{\longrightarrow}\left(q_{1}, \tilde{\sigma_{1}}\right)$ in $B$.

By points 3 and 4, we have: $\left(q_{1}, \overline{\sigma_{1}}\right) \stackrel{v_{2} \mid w_{2}}{\longrightarrow}\left(q_{2}, \overline{\sigma_{2}}\right)$ in $B$.

By points 5 and 6, we have: $\left(q_{2}, \hat{\sigma_{2}}\right) \stackrel{v_{3} \mid w_{3}}{\longrightarrow}\left(q^{\prime}, \hat{\sigma}^{\prime}\right)$ in $B$.

As the underlying input word is cur, observe that we have $\operatorname{MAX}-\operatorname{BAL}(\rho)=\max (n, m)$. Let $\sigma_{0} \in \Gamma_{B}^{\leq K-\operatorname{MAX}-\operatorname{BAL}(\rho)}$. In particular, we have $\left|\sigma_{0}\right| \leq K-n=K-|\sigma|$.

Observe that:

1. as $\mathrm{O}\left(v_{1}\right)=\left(n_{1}, m_{1}\right)$, we have $\left|\tilde{\sigma_{1}}\right| \leq \mathrm{O}_{\max }^{A}$

2. we have $\left|\sigma_{\perp}\right| \leq|\sigma|$ and thus $\left|\sigma_{0} \sigma_{\perp}\right| \leq K$

3. from the two previous points we deduce $\left|\sigma_{0} \sigma_{1}\right| \leq \mathrm{O}_{\max }^{A}+K$, which implies $\left|\sigma_{0} \overline{\sigma_{\perp}}\right| \leq \mathrm{O}_{\max }^{A}+K$

4. as $\mathrm{O}\left(v_{2}\right)=\left(n_{2}, m_{2}\right)$, we have $\left|\overline{\sigma_{1}}\right| \leq K$

From these points we deduce the existence of the following call transition in $C$ :

$$
\left(p, q, \sigma_{0} \sigma\right) \stackrel{c \mid w_{1},\left(\gamma, \sigma_{0} \overline{\sigma_{\perp}}\right)}{\longrightarrow}\left(p_{1}, q_{1}, \overline{\sigma_{1}}\right)
$$

By induction hypothesis applied on $u$, we have that the following run exists in $C$ :

$$
\left(\left(p_{1}, q_{1}, \overline{\sigma_{1}}\right), \perp\right) \stackrel{u \mid w_{2}}{\longrightarrow}\left(\left(p_{2}, q_{2}, \overline{\sigma_{2}}\right), \perp\right)
$$

As above, we have $\left|\sigma_{0}\right| \leq K-m=K-\left|\sigma^{\prime}\right|$. Observe also that as $\mathrm{O}\left(v_{2}\right)=$ $\left(n_{2}, m_{2}\right)$, we have $\left|\overline{\sigma_{2}}\right| \leq K$.

From these points we deduce the existence of the following return transition in $C$ :

$$
\left(p_{2}, q_{2}, \overline{\sigma_{2}}\right) \stackrel{r \mid w_{3},\left(\gamma, \sigma_{0} \overline{\sigma_{\perp}}\right)}{\longrightarrow}\left(p^{\prime}, q^{\prime}, \sigma_{0} \sigma^{\prime}\right)
$$

Using the run of $C$ on $u$, the call transition and the return transition, we can build a run of $C$ on cur as expected. This concludes this proof.

The next lemma states the result in the other way: a run in $C$ implies the existence of corresponding runs in $A$ and $B$. 
Lemma 8. If there exists a run $((p, q, \sigma), \perp) \stackrel{u \mid w}{\longrightarrow}\left(\left(p^{\prime}, q^{\prime}, \sigma^{\prime}\right), \perp\right)$ in $C$, then there exists a word $v \in \Delta^{*}$ and a stack $\sigma_{0} \in \Gamma_{B}^{*}$ such that:

- there exists a run $\rho:(p, \perp) \stackrel{u \mid v}{\longrightarrow}\left(p^{\prime}, \perp\right)$ in $A$

- $\sigma=\sigma_{0} \sigma_{1}, \sigma^{\prime}=\sigma_{0} \sigma_{2}$, and $\mathrm{O}(v)=\left(\left|\sigma_{1}\right|,\left|\sigma_{2}\right|\right)$

- there exists a run $\left(q, \sigma_{1}\right) \stackrel{v \mid w}{\longrightarrow}\left(q^{\prime}, \sigma_{2}\right)$ in $B$

Proof. We proceed by induction on $u \in \Sigma_{\mathrm{wn}}^{*}$.

Case $u=\epsilon, a \in \Sigma_{i}$ : the result directly follows from the definition of $C$.

Case $u=u_{1} u_{2}$. We have a run $(p, q, \sigma) \stackrel{u_{1} \mid w_{1}}{\longrightarrow}\left(p_{1}, q_{1}, \sigma_{1}\right) \stackrel{u_{1} \mid w_{2}}{\longrightarrow}\left(p^{\prime}, q^{\prime}, \sigma^{\prime}\right)$.

By induction hypothesis on $u_{1}$ and $u_{2}$, there exist words $v_{1}, v_{2} \in \Delta^{*}$ and stacks $\sigma_{0}, \sigma_{0}^{\prime}$ such that:

- $\sigma=\sigma_{0} \bar{\sigma}$,

$-\sigma_{1}=\sigma_{0} \overline{\sigma_{1}}$,

- $\sigma_{1}=\sigma_{0}^{\prime} \tilde{\sigma}_{1}$, and

$-\sigma^{\prime}=\sigma_{0}^{\prime} \tilde{\sigma}^{\prime}$.

In addition, the following runs exist in $C$ :

$$
\begin{aligned}
& \text { - }(q, \bar{\sigma}) \stackrel{v_{1} \mid w_{1}}{\longrightarrow}\left(q_{1}, \overline{\sigma_{1}}\right) \\
& \text { - }\left(q_{1}, \tilde{\sigma}_{1}\right) \stackrel{v_{2} \mid w_{2}}{\longrightarrow}\left(q^{\prime}, \tilde{\sigma}^{\prime}\right)
\end{aligned}
$$

It is then easy to prove the result by considering the word $v=v_{1} v_{2}$ and the stack $\sigma_{0}^{\prime \prime}$ such that $\left|\sigma_{0}^{\prime \prime}\right|=\min \left(\left|\sigma_{0}\right|,\left|\sigma_{0}^{\prime}\right|\right)$.

Case $u=c u_{1} r$.

We decompose the run as follows :

$((p, q, \sigma), \perp) \stackrel{c \mid w_{0}}{\longrightarrow}\left(\left(p_{1}, q_{1}, \sigma_{1}\right),\left(\gamma, \sigma_{0}\right)\right) \stackrel{u_{1} \mid w_{1}}{\longrightarrow}\left(\left(p_{2}, q_{2}, \sigma_{2}\right),\left(\gamma, \sigma_{0}\right)\right) \stackrel{r \mid w_{2}}{\longrightarrow}\left(\left(p^{\prime}, q^{\prime}, \sigma^{\prime}\right), \perp\right)$

By definition of call and return transitions of $C$, there exist words $v_{0}, v_{2} \in \Delta^{*}$ such that we have:

- $p \stackrel{c \mid v_{0}, \gamma}{\longrightarrow} p_{1}$ is a call transition of $A$

- $p_{2} \stackrel{r \mid v_{2}, \gamma}{\longrightarrow} p^{\prime}$ is a return transition of $A$

- $(q, \sigma) \stackrel{v_{0} \mid w_{0}}{\longrightarrow}\left(q_{1}, \sigma_{0} \sigma_{1}\right)$ is a run of $B$

- $\left(q_{2}, \sigma_{0} \sigma_{2}\right) \stackrel{v_{2} \mid w_{2}}{\longrightarrow}\left(q^{\prime}, \sigma^{\prime}\right)$ is a run of $B$

By induction hypothesis applied on $u_{1}$, there exist a word $v_{1} \in \Delta^{*}$ and a stack $\sigma_{0}^{\prime} \in \Gamma_{B}^{*}$ such that:

- there exists a run $\left(p_{1}, \perp\right) \stackrel{u_{1} \mid v_{1}}{\longrightarrow}\left(p_{2}, \perp\right)$ in $A$,

- $\sigma_{1}=\sigma_{0}^{\prime} \sigma_{1}^{\prime}$ and $\sigma_{2}=\sigma_{0}^{\prime} \sigma_{2}^{\prime}$,

- there exists a run $\left(q_{1}, \sigma_{1}^{\prime}\right) \stackrel{v_{1} \mid w_{1}}{\longrightarrow}\left(q_{2}, \sigma_{2}^{\prime}\right)$ in $B$. 
Let $v=v_{0} v_{1} v_{2}$. By the previous facts, there exists a run $(p, \perp) \stackrel{u \mid v}{\longrightarrow}\left(p^{\prime}, \perp\right)$ in $A$ and the following run exists in $B$ :

$$
(q, \sigma) \stackrel{v_{0} \mid w_{0}}{\longrightarrow}\left(q_{1}, \sigma_{0} \sigma_{1}\right) \stackrel{v_{1} \mid w_{1}}{\longrightarrow}\left(q_{2}, \sigma_{0} \sigma_{2}\right) \stackrel{v_{2} \mid w_{2}}{\longrightarrow}\left(q^{\prime}, \sigma^{\prime}\right)
$$

Considering $\overline{\sigma_{0}}, \bar{\sigma}, \bar{\sigma}^{\prime}$ such that $\sigma=\overline{\sigma_{0} \sigma}, \sigma^{\prime}={\overline{\sigma_{0}}}^{\prime}$ and $\mathrm{O}(v)=\left(|\bar{\sigma}|,\left|\bar{\sigma}^{\prime}\right|\right)$, we deduce that the following run exists in $C$, which yields the result:

$$
(q, \bar{\sigma}) \stackrel{v \mid w}{\longrightarrow}\left(q^{\prime}, \bar{\sigma}^{\prime}\right)
$$

Theorem 6 follows from Lemma 7 and 8 and from the definition of initial and final states. 